\title{
An Approach to Multicriteria Group Decision-Making with Unknown Weight Information Based on Pythagorean Fuzzy Uncertain Linguistic Aggregation Operators
}

\author{
Chao Liu, ${ }^{1,2}$ Guolin Tang, ${ }^{1,2}$ and Peide Liu $^{3}$ \\ ${ }^{1}$ College of Economics and Management, Beijing University of Technology, Beijing 100124, China \\ ${ }^{2}$ Research Base of Beijing Modern Manufacturing Development, Beijing 100124, China \\ ${ }^{3}$ School of Management Science and Engineering, Shandong University of Finance and Economics, Shandong 250014, China \\ Correspondence should be addressed to Chao Liu; xtkx2011@163.com
}

Received 5 August 2016; Revised 18 December 2016; Accepted 29 December 2016; Published 21 February 2017

Academic Editor: Anna M. Gil-Lafuente

Copyright (C) 2017 Chao Liu et al. This is an open access article distributed under the Creative Commons Attribution License, which permits unrestricted use, distribution, and reproduction in any medium, provided the original work is properly cited.

\begin{abstract}
With respect to multicriteria group decision-making (MCGDM) problems in which the experts have different priority levels, the criteria values are in the form of Pythagorean fuzzy uncertain linguistic variables (PFULVs), and the information about weights of experts and criteria is completely unknown, a novel decision-making method is developed. Firstly, the concept of PFULV is defined, and some operational laws, score function, accuracy function, and normalized Hamming distance of PFULVs are presented. Then, to aggregate information given by all experts, the Pythagorean fuzzy uncertain linguistic prioritized weighted averaging aggregation (PFULPWAA) operator and the Pythagorean fuzzy uncertain linguistic prioritized weighted geometric aggregation (PFULPWGA) operator are proposed. Furthermore, in order to get a comprehensive evaluation value for each alternative, the Pythagorean fuzzy uncertain linguistic Maclaurin symmetric mean aggregation (PFULMSMA) operator and the weighted PFULMSMA (WPFULMSMA) operator are proposed. Moreover, to obtain the information about the weights of criteria, the model based on grey relational analysis (GRA) method is established. Finally, a method of MCGDM with PFULVs is developed, and an application example is given to illustrate the validity and feasibility of the provided procedure.
\end{abstract}

\section{Introduction}

The notion of intuitionistic fuzzy set (IFS) was presented by Atanassov [1], which consists of a membership degree and a nonmembership degree meeting the restriction that the sum of two degrees is equal to or less than 1 . Thus, it is an effective tool to handle uncertainty and vagueness and MCGDM problems with intuitionistic fuzzy numbers (IFNs) have received more and more attention $[2,3]$. Chen et al. [4] defined a new similarity measure between IFSs so as to solve pattern recognition problems. Based on GRA method and evidence theory, Qiu et al. [5] proposed a novel approach to the MCGDM problems in which both the criteria weights and criteria values take the form of IFNs. Montajabiha [6] developed a new version of the PROMETHE II method to solve intuitionistic fuzzy MCGDM problem. He et al. [7] extended power averaging operator, which can reflect the relationship between the arguments being aggregated, to IFS.
He et al. [8] proposed some neutral aggregation operators for IFS, which reflect the interactions between IFNs and the attitude of the experts, and applied them to the MCGDM problem.

However, in some MCGDM problems, the sum of the membership degree and the nonmembership degree to which an alternative satisfies a criterion is bigger than 1, but their square sum is equal to or less than 1 . Thus, the notion of intuitionistic fuzzy set of second type was presented by Atanassov $[9,10]$. Later, the notion of Pythagorean fuzzy set (PFS) was presented by Yager [11, 12], which consists of a membership degree and a nonmembership degree, whose sum of squares is equal to or less than 1. Zhang [13] developed a novel decision method based on similarity measure to deal with selection problem of photovoltaic cells with Pythagorean fuzzy numbers (PFNs). Zhang and Xu [14] gave an extension of TOPSIS method to solve the MCGDM problems under Pythagorean fuzzy environment. Based on 
prospect theory, Ren et al. [15] extended TODIM method to PFS and developed an extended TODIM method. Peng and Yang [16] extended Choquet integral, which can consider the interactions among the criteria, to PFS, and proposed several Pythagorean fuzzy Choquet integral operators.

Under many conditions, it is difficult to handle the fuzziness and uncertainty in real MCGDM problems by numerical numbers, especially for qualitative aspects, while it is easy to express the evaluation values by means of linguistic variables (LVs) or uncertain linguistic variables (ULVs). For example, when the moral character of students, the computer performance, and so on are evaluated, they are easy to be described by the LVs, such as poor, fair, and very good. On the basis of given functions satisfying certain characteristics and distance measures, Tao et al. [17] defined two groups of entropies for LVs and ULVs, respectively. Liu et al. [18] extended Heronian mean (HM) operator, which can consider the interrelationship of the aggregated arguments, to uncertain linguistic set (ULS). Wei et al. [19] extended Bonferroni mean (BM) operator, which can also reflect the interrelationship of the aggregated arguments, to ULS, and applied them to the MCGDM problem with ULVs.

By combining ULVs with IFS, the concept of intuitionistic uncertain linguistic set (IULS) was introduced by Liu and Jin [20], which gives the information about the membership and nonmembership of an element to an ULV. Then, the research on the MCGDM problems with intuitionistic uncertain linguistic numbers (IULNs) has made many achievements [21-23]. However, the IULS cannot handle the situation; the sum of membership degree and nonmembership degree belonging to uncertain linguistic variable is bigger than 1 . To deal with this situation, based on ULVs and PFS, the concept of Pythagorean fuzzy uncertain linguistic set (PFULS), which is only required to meet the restriction that the square sum of the two degrees is less than or equal to 1 , is defined in this paper. To understand the PFULS better, we provide an instance: computer performance is perhaps felt to be lower than "very good" $\left(s_{6}\right)$ but higher than "fair" $\left(s_{3}\right)$, the membership degree to $\left[s_{3}, s_{6}\right]$ is $1 / 2$, and nonmembership degree is $\sqrt{3} / 2$. The evaluation result can be denoted as $\left\langle\left[s_{3}, s_{6}\right],(1 / 2, \sqrt{3} / 2)\right\rangle$. Due to the fact that the sum of two degrees is $(\sqrt{3} / 2+1 / 2)>1$, the evaluation value is not available for IULS but is available for PFULS since $(\sqrt{3} / 2)^{2}+$ $(1 / 2)^{2}=1$. Clearly, the PFULS has more powerful ability than the IULS to depict the uncertainty in the real-world MCGDM problems. It should be noted that when the upper and lower limits of the uncertain linguistic part of PFULS are identical, PFULS reduces to the Pythagorean fuzzy linguistic set (PFLS) introduced by Peng and Yang [24], which indicates that the former is an extension of the latter. Compared with PFLS, PFULS is defined by utilizing ULVs, whose membership degree and nonmembership degree are no longer with respect to a LV, but to an ULV, which makes the experts express uncertain information more easily and precisely.

Information aggregation is a pervasive activity in our daily life; many operators have been provided on this issue. Among them, the prioritized averaging (PA) operator and the Maclaurin symmetric mean (MSM) are two of the most common operators for aggregating information. The PA operator was initially presented by Yager [25], which can capture the prioritization phenomenon of the aggregated arguments. Then, it was extended to hesitant fuzzy set (HFS) [26], triangular fuzzy set (TFS) [27], IFS [28], trapezoidal intuitionistic fuzzy set (TIFS) [29], linguistic set (LS) [30], 2-tuple linguistic set (2TLS) [31], multigranular uncertain linguistic set (MULS) [32], and so on. The MSM was initially given by Maclaurin [33], which can consider the interdependent characteristics among the multi-input arguments. The MSM is different from Choquet integral or power average operator. The MSM pays attention to the input arguments while the Choquet integral or power average operator pays attention to the weights information. The MSM is also different form BM or HM. The MSM operator considers the interdependent characteristics among the multi-input arguments and should take one parameter from finite integer set, while the BM or HM captures the interrelationship between two input arguments and should take two parameters both from infinite set. Due to the advantages of the MSM, it was extended to HFS [34], IFS [3], 2TLS [35], ULS [36], intuitionistic linguistic set (ILS) [21], IULS [21], and so on. However, both PA and MSM operators fail to aggregate Pythagorean fuzzy uncertain linguistic information. Therefore, we shall propose the PFULPWAA, PFULPWGA, PFULMSMA, and WPFULMSMA operators. The significant features of these operators are that not only can they handle PFULVs, but also the PFULPWAA and PFULPWGA operators can capture prioritization among the criteria or experts, and the PFULMSMA and WPFULMSMA operators can consider the interrelationship among the multi-input arguments.

The aim of this paper is to develop a novel method based on proposed Pythagorean fuzzy uncertain linguistic aggregation operators to solve the MCGDM problems in which the experts have different priority levels, the criteria values are in the form of PFULVs, and the information about weights of experts and criteria is completely unknown. To do so, the remainder of this paper is constructed as follows: Section 2 reviews some concepts of PFS, PA operator, and MSM. Section 3 defines the concept of PFULS and presents some operational laws, score function, accuracy function, and normalized Hamming distance of PFULVs. Section 4 proposes the PFULPWAA and PFULPWGA operators and investigates their corresponding properties. Section 5 proposes the PFULMSMA and WPFULMSMA operators. Section 6 presents an approach to Pythagorean fuzzy linguistic MCGDM based on GRA model and the proposed new operators. Section 7 provides an example to demonstrate the decision-making application. Section 8 gives the concluding remarks.

\section{Preliminary}

\subsection{The Pythagorean Fuzzy Set}

Definition 1 (see [11]). Suppose $X=\left\{x_{i}\right\}(i=1,2, \ldots, n)$ is a universe of discourse. A Pythagorean fuzzy set (PFS) $P$ in $X$ is an expression given by

$$
P=\left\{\left\langle x_{i}, u_{p}\left(x_{i}\right), v_{p}\left(x_{i}\right)\right\rangle \mid x_{i} \in X\right\},
$$


where the functions $u_{p}\left(x_{i}\right): X \rightarrow[0,1]$ and $v_{p}\left(x_{i}\right): X \rightarrow$ $[0,1]$ are the degree of membership and the degree of nonmembership of the element $x_{i} \in X$ to the set $P$, respectively, meeting the condition that $0 \leq\left(u_{p}\left(x_{i}\right)\right)^{2}+\left(v_{p}\left(x_{i}\right)\right)^{2} \leq 1$. The function $\pi_{p}\left(x_{i}\right)=\sqrt{1-\left(u_{p}\left(x_{i}\right)\right)^{2}-\left(v_{p}\left(x_{i}\right)\right)^{2}}$ is called the degree of indeterminacy of $x_{i} \in X$ to the set $P$. For computational convenience, $p\left(x_{i}\right)=\left(u_{p}\left(x_{i}\right), v_{p}\left(x_{i}\right)\right)$ is called a Pythagorean fuzzy number (PFN) represented by $p_{i}=$ $\left(u_{i}, v_{i}\right)[14]$.

With respect to the operational rules and characteristics of PFNs, please refer to [11, 14].

2.2. The Prioritized Averaging Operator. In many real and practical MCGDM problems, the criteria or the experts usually have different priority levels. For instance, regarding decision-making in a company, general manager usually has a higher priority than vice manager. To deal with this issue, the prioritized averaging (PA) operator was proposed by Yager [25], which is shown as follows.

Definition 2 (see [25]). Suppose that the criteria $C=\left\{c_{1}, c_{2}\right.$, $\left.\ldots, c_{n}\right\}$ are prioritized, where $c_{1}>c_{2}>\cdots>c_{n}$ (" $>$ " denotes "be superior to"). The value $c_{l}(a)$ is the criterion value of any alternative $a$ under criterion $c_{l}$ and meets $c_{l}(a) \in[0,1]$. If

$$
\operatorname{PA}\left(c_{l}(a)\right)=\sum_{l=1}^{n} w_{l} c_{l}(a)
$$

where $w_{l}=T_{l} / \sum_{l=1}^{n} T_{l}, T_{l}=\prod_{j=1}^{l-1} c_{j}(a)(l=2,3, \ldots, n), T_{1}=$ 1. Then, PA is called the prioritized averaging operator.

2.3. The Maclaurin Symmetric Mean. The MSM can consider the interdependent characteristics among the multi-input arguments, which is shown as follows.

Definition 3 (see [33]). Suppose $B=\left\{b_{1}, b_{2}, \ldots, b_{n}\right\}$ is a collection of nonnegative real numbers, and $\kappa=1,2, \ldots, n$. If

$$
\operatorname{MSM}^{(\kappa)}\left(\alpha_{1}, \alpha_{2}, \ldots, \alpha_{n}\right)=\left(\frac{\sum_{1 \leq l_{1}<\cdots<l_{\kappa} \leq n} \prod_{j=1}^{\kappa} b_{l_{j}}}{C_{n}^{\kappa}}\right)^{1 / \kappa}
$$

where $C_{n}^{\kappa}$ is the binomial coefficient, and $\left\{l_{1}, l_{2}, \ldots, l_{\kappa}\right\}$ traverses all the $k$-tuple combinations of $\{1,2, \ldots, n\}$, then, $\mathrm{MSM}^{(k)}$ is called the Maclaurin symmetric mean.

\section{Pythagorean Fuzzy Uncertain Linguistic Variable}

3.1. The Pythagorean Fuzzy Uncertain Linguistic Set. Motivated by PFLS [24] that is based on LVs and PFS, we define the notion of PFULS based on ULVs and PFS and present operational laws, score function, accuracy function, and normalized Hamming distance of PFULVs.
Definition 4. Suppose $X$ is a universe of discourse. A PFULS $\widetilde{P}$ in $X$ is an expression given by

$$
\widetilde{P}=\left\{\left\langle x_{i},\left[s_{\theta\left(x_{i}\right)}, s_{\tau\left(x_{i}\right)}\right],\left(u_{\tilde{p}}\left(x_{i}\right), v_{\tilde{p}}\left(x_{i}\right)\right)\right\rangle \mid x_{i} \in X\right\},
$$

where $\left[s_{\theta\left(x_{i}\right)}, s_{\tau\left(x_{i}\right)}\right]$ is an ULV defined by $\mathrm{Xu}$ [37] and $\left(u_{\tilde{p}}\left(x_{i}\right), v_{\tilde{p}}\left(x_{i}\right)\right)$ is a PFS expressed in Definition 1 which denotes the degrees of the element $x_{i} \in X$ to the ULV $\left[s_{\theta\left(x_{i}\right)}, s_{\tau\left(x_{i}\right)}\right]$. The function $\pi_{\tilde{p}}\left(x_{i}\right)=$ $\sqrt{1-\left(u_{\tilde{p}}\left(x_{i}\right)\right)^{2}-\left(v_{\tilde{p}}\left(x_{i}\right)\right)^{2}}$ is called the degree of indeterminacy of $x_{i} \in X$ to the ULV $\left[s_{\theta\left(x_{i}\right)}, s_{\tau\left(x_{i}\right)}\right]$. For computational convenience, $\widetilde{p}\left(x_{i}\right)=\left\langle\left[s_{\theta\left(x_{i}\right)}, s_{\tau\left(x_{i}\right)}\right],\left(u_{\tilde{p}}\left(x_{i}\right), v_{\tilde{p}}\left(x_{i}\right)\right)\right\rangle$ is called a Pythagorean fuzzy linguistic variable (PFULV) represented by $\widetilde{p}_{i}=\left\langle\left[s_{\theta_{i}}, s_{\tau_{i}}\right],\left(u_{i}, v_{i}\right)\right\rangle$.

Suppose that $\widetilde{p}_{1}=\left\langle\left[s_{\theta_{1}}, s_{\tau_{1}}\right],\left(u_{1}, v_{1}\right)\right\rangle$ and $\widetilde{p}_{2}=\left\langle\left[s_{\theta_{2}}\right.\right.$, $\left.\left.s_{\tau_{2}}\right],\left(u_{2}, v_{2}\right)\right\rangle$ are any two PFULVs and $\lambda \geq 0$; then based on operational laws of ULVs and PFNs, the operational laws of $\widetilde{p}_{1}$ and $\widetilde{p}_{2}$ are given as follows:

(1) $\widetilde{p}_{1} \oplus \widetilde{p}_{2}$

$$
=\left\langle\left[s_{\theta_{1}+\theta_{2}}, s_{\tau_{1}+\tau_{2}}\right],\left(\sqrt{u_{1}^{2}+u_{2}^{2}-u_{1}^{2} u_{2}^{2}}, v_{1} v_{2}\right)\right\rangle,
$$

(2) $\widetilde{p}_{1} \otimes \widetilde{p}_{2}$

$$
=\left\langle\left[s_{\theta_{1} \cdot \theta_{2}}, s_{\tau_{1} \cdot \tau_{2}}\right],\left(u_{1} u_{2}, \sqrt{v_{1}^{2}+v_{2}^{2}-v_{1}^{2} v_{2}^{2}}\right)\right\rangle,
$$

(3) $\lambda \widetilde{p}_{1}=\left[s_{\lambda \cdot \theta_{1}}, s_{\lambda \cdot \tau_{1}}\right],\left(\sqrt{1-\left(1-u_{1}^{2}\right)^{\lambda}}, v_{1}^{\lambda}\right)$,

(4) $\left(\tilde{p}_{1}\right)^{\lambda}=\left\langle\left[s_{\theta_{1} \lambda}, s_{\tau_{1}{ }^{\lambda}}\right],\left(u_{1}^{\lambda}, \sqrt{1-\left(1-v_{1}^{2}\right)^{\lambda}}\right)\right\rangle$.

Clearly, the above operational results are still PFULVs.

Theorem 5. Suppose that $\tilde{p}_{1}=\left\langle\left[s_{\theta_{1}}, s_{\tau_{1}}\right],\left(u_{1}, v_{1}\right)\right\rangle$ and $\tilde{p}_{2}=$ $\left\langle\left[s_{\theta_{2}}, s_{\tau_{2}}\right],\left(u_{2}, v_{2}\right)\right\rangle$ are any two PFULVs; then we can get the following calculation rules:
(1) $\widetilde{p}_{1} \oplus \widetilde{p}_{2}=\widetilde{p}_{2} \oplus \widetilde{p}_{1}$
(2) $\widetilde{p}_{1} \otimes \widetilde{p}_{2}=\widetilde{p}_{2} \otimes \widetilde{p}_{1}$,
(3) $\lambda\left(\widetilde{p}_{1} \oplus \tilde{p}_{2}\right)=\lambda \widetilde{p}_{1} \oplus \lambda \widetilde{p}_{2}$,
(4) $\tilde{p}_{1}^{\lambda} \otimes \tilde{p}_{2}^{\lambda}=\left(\tilde{p}_{1} \otimes \tilde{p}_{2}\right)^{\lambda}$,
(5) $\lambda_{1} \widetilde{p}_{1} \oplus \lambda_{2} \widetilde{p}_{1}=\left(\lambda_{1}+\lambda_{2}\right) \widetilde{p}_{1}$,
(6) $\widetilde{p}_{1}^{\lambda_{1}} \otimes \widetilde{p}_{1}^{\lambda_{2}}=\widetilde{p}_{1}^{\lambda_{1}+\lambda_{2}}$.

Proof. (1) According to formulas (5) and (6), formulas (9) and (10) are right. 
(2) For formula (11),

$$
\begin{aligned}
& \lambda\left(\widetilde{p}_{1} \oplus \tilde{p}_{2}\right)=\lambda\left\langle\left[s_{\theta_{1}+\theta_{2}}, s_{\tau_{1}+\tau_{2}}\right],\right. \\
& \left.\left(\sqrt{u_{1}^{2}+u_{2}^{2}-u_{1}^{2} u_{2}^{2}}, v_{1} v_{2}\right)\right\rangle=\left\langle\left[s_{\lambda\left(\theta_{1}+\theta_{2}\right)}, s_{\lambda\left(\tau_{1}+\tau_{2}\right)}\right]\right. \text {, } \\
& \left.\left(\sqrt{1-\left(1-u_{1}^{2}-u_{2}^{2}+u_{1}^{2} u_{2}^{2}\right)^{\lambda}},\left(v_{1} v_{2}\right)^{\lambda}\right)\right\rangle \\
& =\left\langle\left[s_{\lambda \cdot \theta_{1}+\lambda \cdot \theta_{2}}, s_{\lambda \cdot \tau_{1}+\lambda \cdot \tau_{2}}\right],\right. \\
& \left.\left(\sqrt{1-\left(1-u_{1}^{2}\right)^{\lambda}\left(1-u_{2}^{2}\right)^{\lambda}}, v_{1}^{\lambda} v_{2}^{\lambda}\right)\right\rangle=\left\langle\left[s_{\lambda \cdot \theta_{1}}, s_{\lambda \cdot \tau_{1}}\right],\right. \\
& \left.\left(\sqrt{1-\left(1-u_{1}^{2}\right)^{\lambda}}, v_{1}^{\lambda}\right)\right\rangle \oplus\left\langle\left[s_{\lambda \cdot \theta_{2}}, s_{\lambda \cdot \tau_{2}}\right],\right. \\
& \left.\left(\sqrt{1-\left(1-u_{2}^{2}\right)^{\lambda}}, v_{2}^{\lambda}\right)\right\rangle=\lambda\left\langle\left[s_{\theta_{1}}, s_{\tau_{1}}\right],\left(u_{1}, v_{1}\right)\right\rangle \\
& \oplus \lambda\left\langle\left[s_{\theta_{2}}, s_{\tau_{2}}\right],\left(u_{2}, v_{2}\right)\right\rangle=\lambda \widetilde{p}_{1} \oplus \lambda \tilde{p}_{2}
\end{aligned}
$$

and the proof of formula (11) is finished.

(3) Similar to proof of formula (11), it can be easily proved that formulas (12), (13), and (14) are right.

\subsection{Comparison of Two Pythagorean Fuzzy Uncertain Linguistic Variables}

Definition 6. Suppose that $\widetilde{p}_{1}=\left\langle\left[s_{\theta_{1}}, s_{\tau_{1}}\right],\left(u_{1}, v_{1}\right)\right\rangle$ is a PFULV; then the score function of $\widetilde{p}_{1}$ is shown as follows:

$$
S^{\prime}\left(\widetilde{p}_{1}\right)=\frac{\theta_{1}+\tau_{1}}{2(t-1)}\left(u_{1}^{2}-v_{1}^{2}\right)
$$

It should be mentioned that the score function $S^{\prime}\left(\widetilde{p}_{1}\right)$ is between -1 and 1 . In order to facilitate the following study, we provide another score function $S^{\prime}\left(\widetilde{p}_{1}\right)$ whose range is between 0 and 1 .

$$
S\left(\widetilde{p}_{1}\right)=\frac{\theta_{1}+\tau_{1}}{4(t-1)}\left(u_{1}^{2}+1-v_{1}^{2}\right)
$$

Definition 7. Suppose that $\widetilde{p}_{1}=\left\langle\left[s_{\theta_{1}}, s_{\tau_{1}}\right],\left(u_{1}, v_{1}\right)\right\rangle$ is a PFULV; then the accuracy function of $\widetilde{p}_{1}$ is shown as follows:

$$
A\left(\widetilde{p}_{1}\right)=\frac{\theta_{1}+\tau_{1}}{2(t-1)}\left(u_{1}^{2}+v_{1}^{2}\right) .
$$

Definition 8. Suppose that $\tilde{p}_{1}=\left\langle\left[s_{\theta_{1}}, s_{\tau_{1}}\right],\left(u_{1}, v_{1}\right)\right\rangle$ and $\widetilde{p}_{2}=$ $\left\langle\left[s_{\theta_{2}}, s_{\tau_{2}}\right],\left(u_{2}, v_{2}\right)\right\rangle$ are any two PFULVs; then their comparison rules are shown as follows:

(1) If $S\left(\widetilde{p}_{1}\right)>S\left(\widetilde{p}_{2}\right)$, then $\widetilde{p}_{1}>\widetilde{p}_{2}$.

(2) If $S\left(\widetilde{p}_{1}\right)=S\left(\widetilde{p}_{2}\right)$ and $A\left(\widetilde{p}_{1}\right)>A\left(\widetilde{p}_{2}\right)$, then $\widetilde{p}_{1}>\widetilde{p}_{2}$.

(3) If $S\left(\widetilde{p}_{1}\right)=S\left(\widetilde{p}_{2}\right)$ and $A\left(\widetilde{p}_{1}\right)=A\left(\widetilde{p}_{2}\right)$, then $\widetilde{p}_{1}=\widetilde{p}_{2}$.

\subsection{The Normalized Hamming Distance between Two PFULVs}

Definition 9. Let $\widetilde{p}_{1}=\left\langle\left[s_{\theta_{1}}, s_{\tau_{1}}\right],\left(u_{1}, v_{1}\right)\right\rangle, \widetilde{p}_{2}=\left\langle\left[s_{\theta_{2}}, s_{\tau_{2}}\right]\right.$, $\left.\left(u_{2}, v_{2}\right)\right\rangle$, and $\widetilde{p}_{3}=\left\langle\left[s_{\theta_{3}}, s_{\tau_{3}}\right],\left(u_{3}, v_{3}\right)\right\rangle$ be any three PFULVs. If $d\left(\widetilde{p}_{1}, \widetilde{p}_{2}\right)$ satisfies the three restrictions

(1) $0 \leq d\left(\widetilde{p}_{1}, \widetilde{p}_{2}\right) \leq 1, d\left(\widetilde{p}_{1}, \widetilde{p}_{1}\right)=0$,

(2) $d\left(\widetilde{p}_{2}, \widetilde{p}_{1}\right)=d\left(\widetilde{p}_{1}, \widetilde{p}_{2}\right)$,

(3) $d\left(\tilde{p}_{1}, \widetilde{p}_{2}\right)+d\left(\tilde{p}_{2}, \tilde{p}_{3}\right) \geq d\left(\tilde{p}_{1}, \widetilde{p}_{3}\right)$,

then $d\left(\widetilde{p}_{1}, \widetilde{p}_{2}\right)$ is called the distance between $\widehat{p}_{1}$ and $\widehat{p}_{2}$.

Definition 10. Suppose that $\widetilde{p}_{1}=\left\langle\left[s_{\theta_{1}}, s_{\tau_{1}}\right],\left(u_{1}, v_{1}\right)\right\rangle$ and $\widetilde{p}_{2}=$ $\left\langle\left[s_{\theta_{2}}, s_{\tau_{2}}\right],\left(u_{2}, v_{2}\right)\right\rangle$ are any two PFULVs; then the normalized Hamming distance $d\left(\widehat{p}_{1}, \widehat{p}_{2}\right)$ between $\widehat{p}_{1}$ and $\widehat{p}_{2}$ is defined as follows:

$$
\begin{aligned}
& d\left(p_{1}, p_{2}\right)=\frac{1}{6(t-1)}\left(\left|\theta_{1} u_{1}^{2}-\theta_{2} u_{2}^{2}\right|+\left|\theta_{1} v_{1}^{2}-\theta_{2} v_{2}^{2}\right|\right. \\
& \quad+\left|\theta_{1} \pi_{1}^{2}-\theta_{2} \pi_{2}^{2}\right|+\left|\tau_{1} u_{1}^{2}-\tau_{2} u_{2}^{2}\right|+\left|\tau_{1} v_{1}^{2}-\tau_{2} v_{2}^{2}\right| \\
& \left.\quad+\left|\tau_{1} \pi_{1}^{2}-\tau_{2} \pi_{2}^{2}\right|\right) .
\end{aligned}
$$

Proof. Clearly, the normalized Hamming distance $d\left(\widehat{p}_{1}, \widehat{p}_{2}\right)$ defined in (19) satisfies restrictions (1) and (2) in Definition 9. In what follows, we shall prove that the normalized Hamming distance $d\left(\widehat{p}_{1}, \widehat{p}_{2}\right)$ defined in (19) can also satisfy restriction (3) in Definition 9.

$$
\begin{aligned}
d & \left(p_{1}, p_{3}\right)=\frac{1}{6(t-1)}\left(\left|\theta_{1} u_{1}^{2}-\theta_{3} u_{3}^{2}\right|+\left|\theta_{1} v_{1}^{2}-\theta_{3} v_{3}^{2}\right|\right. \\
& +\left|\theta_{1} \pi_{1}^{2}-\theta_{3} \pi_{3}^{2}\right|+\left|\tau_{1} u_{1}^{2}-\tau_{3} u_{3}^{2}\right|+\left|\tau_{1} v_{1}^{2}-\tau_{3} v_{3}^{2}\right| \\
& \left.+\left|\tau_{1} \pi_{1}^{2}-\tau_{3} \pi_{3}^{2}\right|\right) \\
& =\frac{1}{6(t-1)}\left(\left|\theta_{1} u_{1}^{2}-\theta_{2} u_{2}^{2}+\theta_{2} u_{2}^{2}-\theta_{3} u_{3}^{2}\right|\right. \\
& +\left|\theta_{1} v_{1}^{2}-\theta_{2} v_{2}^{2}+\theta_{2} v_{2}^{2}-\theta_{3} v_{3}^{2}\right| \\
& +\left|\theta_{1} \pi_{1}^{2}-\theta_{2} \pi_{2}^{2}+\theta_{2} \pi_{2}^{2}-\theta_{3} \pi_{3}^{2}\right| \\
& +\left|\tau_{1} u_{1}^{2}-\tau_{2} u_{2}^{2}+\tau_{2} u_{2}^{2}-\tau_{3} u_{3}^{2}\right| \\
& +\left|\tau_{1} v_{1}^{2}-\tau_{2} v_{2}^{2}+\tau_{2} v_{2}^{2}-\tau_{3} v_{3}^{2}\right| \\
& \left.+\left|\tau_{1} \pi_{1}^{2}-\tau_{2} \pi_{2}^{2}+\tau_{2} \pi_{2}^{2}-\tau_{3} \pi_{3}^{2}\right|\right) \\
& \leq \frac{1}{6(t-1)}\left(\left|\theta_{1} u_{1}^{2}-\theta_{2} u_{2}^{2}\right|+\left|\theta_{2} u_{2}^{2}-\theta_{3} u_{3}^{2}\right|\right. \\
& +\left|\theta_{1} v_{1}^{2}-\theta_{2} v_{2}^{2}\right|+\left|\theta_{2} v_{2}^{2}-\theta_{3} v_{3}^{2}\right|+\left|\theta_{1} \pi_{1}^{2}-\theta_{2} \pi_{2}^{2}\right| \\
& +\left|\theta_{2} \pi_{2}^{2}-\theta_{3} \pi_{3}^{2}\right|+\left|\tau_{1} u_{1}^{2}-\tau_{2} u_{2}^{2}\right|+\left|\tau_{2} u_{2}^{2}-\tau_{3} u_{3}^{2}\right|
\end{aligned}
$$




$$
\begin{aligned}
& +\left|\tau_{1} v_{1}^{2}-\tau_{2} v_{2}^{2}\right|+\left|\tau_{2} v_{2}^{2}-\tau_{3} v_{3}^{2}\right|+\left|\tau_{1} \pi_{1}^{2}-\tau_{2} \pi_{2}^{2}\right| \\
& \left.+\left|\tau_{2} \pi_{2}^{2}-\tau_{3} \pi_{3}^{2}\right|\right)=\frac{1}{6(t-1)}\left(\left|\theta_{1} u_{1}^{2}-\theta_{2} u_{2}^{2}\right|\right. \\
& +\left|\theta_{1} v_{1}^{2}-\theta_{2} v_{2}^{2}\right|+\left|\theta_{1} \pi_{1}^{2}-\theta_{2} \pi_{2}^{2}\right|+\left|\tau_{1} u_{1}^{2}-\tau_{2} u_{2}^{2}\right| \\
& \left.+\left|\tau_{1} v_{1}^{2}-\tau_{2} v_{2}^{2}\right|+\left|\tau_{1} \pi_{1}^{2}-\tau_{2} \pi_{2}^{2}\right|\right) \\
& +\frac{1}{6(t-1)}\left(\left|\theta_{2} u_{2}^{2}-\theta_{3} u_{3}^{2}\right|+\left|\theta_{2} v_{2}^{2}-\theta_{3} v_{3}^{2}\right|\right. \\
& +\left|\theta_{2} \pi_{2}^{2}-\theta_{3} \pi_{3}^{2}\right|+\left|\tau_{2} u_{2}^{2}-\tau_{3} u_{3}^{2}\right|+\left|\tau_{2} v_{2}^{2}-\tau_{3} v_{3}^{2}\right| \\
& \left.+\left|\tau_{2} \pi_{2}^{2}-\tau_{3} \pi_{3}^{2}\right|\right)=d\left(p_{1}, p_{2}\right)+d\left(p_{2}, p_{3}\right)
\end{aligned}
$$

and therefore

$$
d\left(\tilde{p}_{1}, \tilde{p}_{2}\right)+d\left(\widetilde{p}_{2}, \tilde{p}_{3}\right) \geq d\left(\widetilde{p}_{1}, \widetilde{p}_{3}\right) .
$$

\section{Pythagorean Fuzzy Uncertain Linguistic Prioritized Aggregation Operators}

In this section, we shall extend the PA operator to PFULS and propose the PPFULPWAA and PFULPWGA operators.

\subsection{The PFULPWAA Operator}

Definition 11. Suppose $\widetilde{P}$ is the set of all PFULVs, $\widetilde{p}_{1}=$ $\left\langle\left[s_{\theta_{l}}, s_{\tau_{l}}\right],\left(u_{l}, v_{l}\right)\right\rangle(l=1,2, \ldots, n)$ is a collection of PFULVs, and PFULPWAA: $\widetilde{P}^{n} \rightarrow \widetilde{P}$; if

$$
\operatorname{PFULPWAA}\left(\tilde{p}_{1}, \tilde{p}_{2}, \ldots, \widetilde{p}_{n}\right)=\bigoplus_{l=1}^{n}\left(\frac{T_{l}}{\sum_{l=1}^{n} T_{l}} \widetilde{p}_{l}\right),
$$

where $T_{l}=\prod_{j=1}^{l-1} S\left(\widetilde{p}_{j}\right)(l=2,3, \ldots, n), T_{1}=1$, and $S\left(\widetilde{p}_{j}\right)$ is the score value of a PFULV $\widetilde{p}_{j}$, then PFULPWAA is called the Pythagorean fuzzy uncertain linguistic prioritized weighted averaging aggregation operator.

Theorem 12. Suppose that $\tilde{p}_{l}=\left\langle\left[s_{\theta_{l}}, s_{\tau_{l}}\right],\left(u_{l}, v_{l}\right)\right\rangle(l=1,2$, $\ldots, n)$ is a collection of PFULVs; then the aggregating value by PFULPWAA operator is still a PFULV, and

$$
\begin{aligned}
& \bigoplus_{l=1}^{n}\left(\frac{T_{l}}{\sum_{l=1}^{n} T_{l}} \tilde{p}_{l}\right) \\
& =\left\langle\left[s_{\sum_{l=1}^{n}\left(T_{l} / \sum_{l=1}^{n} T_{l}\right) \theta_{l}}, s_{\sum_{l=1}^{n}\left(T_{l} / \sum_{l=1}^{n} T_{l}\right) \tau_{l}}\right],\right.
\end{aligned}
$$

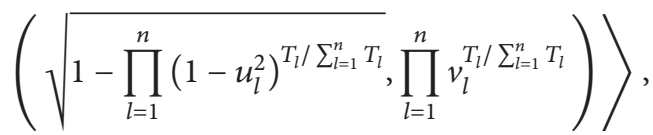

where $T_{l}=\prod_{j=1}^{l-1} S\left(\widetilde{p}_{j}\right)(l=2,3, \ldots, n), T_{1}=1$, and $S\left(\widetilde{p}_{j}\right)$ is the score value of the $\widetilde{p}_{j}$.
Proof. Formula (23) can be proved by mathematical introduction on $n$ as follows.

(i) For $n=2$, according to the operational laws of PFULVs, we obtain

$$
\begin{aligned}
& \frac{T_{j}}{\sum_{l=1}^{n} T_{l}} \widetilde{p}_{j}=\left\langle\left[s_{\left(T_{j} / \sum_{l=1}^{n} T_{l}\right) \theta_{j}}, s_{\left(T_{j} / \sum_{l=1}^{n} T_{l}\right) \tau_{j}}\right],\right. \\
& \quad\left(\sqrt{\left.\left.1-\left(1-u_{j}^{2}\right)^{T_{j} / \sum_{l=1}^{n} T_{l}}, v_{j}^{T_{j} / \sum_{l=1}^{n} T_{l}}\right)\right\rangle \quad(j=1,2),}\right.
\end{aligned}
$$$$
\operatorname{PFULPWAA}\left(\widetilde{p}_{1}, \widetilde{p}_{2}\right)=\left(\frac{T_{1}}{\sum_{l=1}^{n} T_{l}} \widetilde{p}_{1}\right) \oplus\left(\frac{T_{2}}{\sum_{l=1}^{n} T_{l}}\right.
$$

$$
\left.\cdot \widetilde{p}_{2}\right)=\left\langle\left[s_{\sum_{l=1}^{2}\left(T_{l} / \sum_{l=1}^{n} T_{l}\right) \theta_{l}}, s_{\sum_{l=1}^{2}\left(T_{l} / \sum_{l=1}^{n} T_{l}\right) \tau_{l}}\right],\right.
$$

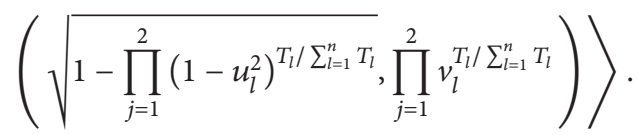

So, formula (23) is right for $n=2$.

(ii) Suppose that formula (23) is also right for $n=k$; that is,

$$
\begin{aligned}
& \operatorname{PFULPWAA}\left(\tilde{p}_{1}, \tilde{p}_{2}, \ldots, \tilde{p}_{k}\right) \\
& \quad=\left\langle\left[ s_{\left.\sum_{l=1}^{k}\left(T_{l} / \sum_{l=1}^{n} T_{l}\right) \theta_{l}, s_{\sum_{l=1}^{k}\left(T_{l} / \sum_{l=1}^{n} T_{l}\right) \tau_{l}}\right],}\right.\right. \\
& \quad\left(\sqrt{\left.\left.1-\prod_{l=1}^{k}\left(1-u_{l}^{2}\right)^{T_{l} / \sum_{l=1}^{n} T_{l}}, \prod_{l=1}^{k} v_{l}^{T_{l} / \sum_{l=1}^{n} T_{l}}\right)\right\rangle .}\right.
\end{aligned}
$$

(iii) For $n=k+1$, we obtain

$$
\begin{aligned}
& \operatorname{PFULPWAA}\left(\tilde{p}_{1}, \tilde{p}_{2}, \ldots, \tilde{p}_{k}, \tilde{p}_{k+1}\right) \\
& =\left\langle\left[s_{\sum_{l=1}^{k}\left(T_{l} / \sum_{l=1}^{n} T_{l}\right) \theta_{l}}, s_{\sum_{l=1}^{k}\left(T_{l} / \sum_{l=1}^{n} T_{l}\right) \tau_{l}}\right],\right. \\
& \left.\left(\sqrt{1-\prod_{l=1}^{k}\left(1-u_{l}^{2}\right)^{T_{l} / \sum_{l=1}^{n} T_{l}}}, \prod_{l=1}^{k} v_{l}^{T_{l} / \sum_{l=1}^{n} T_{l}}\right)\right\rangle \\
& \oplus\left\langle\left[s_{\left(T_{k+1} / \sum_{l=1}^{n} T_{l}\right) \theta_{k+1}}, s_{\left(T_{k+1} / \sum_{l=1}^{n} T_{l}\right) \tau_{k+1}}\right],\right. \\
& \left.\left(\sqrt{1-\left(1-u_{k+1}^{2}\right)^{T_{k+1} / \sum_{l=1}^{n} T_{l}}}, v_{k+1}^{T_{k+1} / \sum_{l=1}^{n} T_{l}}\right)\right\rangle \\
& =\left\langle\left[s_{\sum_{l=1}^{k+1}\left(T_{l} / \sum_{l=1}^{n} T_{j}\right) \theta_{l}} s_{\sum_{l=1}^{k+1}\left(T_{l} / \sum_{l=1}^{n} T_{l}\right) \tau_{l}}\right],\right.
\end{aligned}
$$

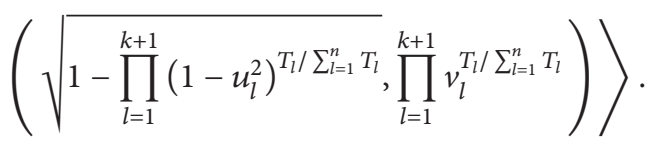


So, formula (23) is right for $n=k+1$. According to (i), (ii), and (iii), formula (23) is right for all $n$.

In addition, since

$$
\begin{aligned}
& 0 \leq \sum_{l=1}^{n} \frac{T_{l}}{\sum_{l=1}^{n} T_{l}} \theta_{l} \leq t-1, \\
& 0 \leq \sum_{l=1}^{n} \frac{T_{l}}{\sum_{l=1}^{n} T_{l}} \tau_{l} \leq t-1, \\
& \sum_{l=1}^{n} \frac{T_{l}}{\sum_{l=1}^{n} T_{l}} \theta_{l} \leq \sum_{l=1}^{n} \frac{T_{l}}{\sum_{l=1}^{n} T_{l}} \tau_{l}, \\
& 0 \leq \sqrt{1-\prod_{l=1}^{n}\left(1-u_{l}^{2}\right)^{T_{l} / \sum_{l=1}^{n} T_{l}}} \leq 1, \\
& 0 \leq \prod_{l=1}^{n} v_{l}^{T_{l} / \sum_{l=1}^{n} T_{l}} \leq 1, \\
& 0 \leq\left(\sqrt{1-\prod_{l=1}^{n}\left(1-u_{l}^{2}\right)^{\sum_{l=1}^{n}\left(T_{l} / \sum_{l=1}^{n} T_{l}\right)}}\right)^{2} \\
& +\left(\prod_{l=1}^{n} v_{l}^{\sum_{l=1}^{n}\left(T_{l} / \sum_{l=1}^{n} T_{l}\right)}\right)^{2} \\
& \leq 1-\prod_{l=1}^{n}\left(1-u_{l}^{2}\right)^{\sum_{l=1}^{n}\left(T_{l} / \sum_{l=1}^{n} T_{l}\right)} \\
& +\prod_{l=1}^{n}\left(1-u_{l}^{2}\right)^{\sum_{l=1}^{n}\left(T_{l} / \sum_{l=1}^{n} T_{l}\right)}=1
\end{aligned}
$$

formula (23) is still a PFULV, and the proof of Theorem 12 is finished.

In what follows, some properties of the PFULPWAA operator shall be explored.

Theorem 13. Suppose that $\widetilde{p}_{l}=\left\langle\left[s_{\theta_{l}}, s_{\tau_{l}}\right],\left(u_{l}, v_{l}\right)\right\rangle(l=1,2$, $\ldots, n)$ is a collection of PFULVs; then the PFULPWAA operator has the following properties:

(1) (Idempotency). Suppose all $\widetilde{p}_{l}=\left\langle\left[s_{\theta_{l}}, s_{\tau_{l}}\right],\left(u_{l}, v_{l}\right)\right\rangle(l=$ $1,2, \ldots, n)$ are equal; that is, $\widetilde{p}_{l}=\widetilde{p}=\left\langle\left[s_{\theta}, s_{\tau}\right],(u\right.$, $v)\rangle(l=1,2, \ldots, n)$, and then

$$
\operatorname{PFULPWAA}\left(\widetilde{p}_{1}, \widetilde{p}_{2}, \ldots, \widetilde{p}_{n}\right)=\tilde{p}
$$

(2) (Boundness). Suppose $\tilde{p}^{+}=\left\langle\left[\max _{l}\left(s_{\theta_{l}}\right), \max _{l}\left(s_{\tau_{l}}\right)\right]\right.$, $\left.\left(\max _{l}\left(u_{l}\right), \min _{l}\left(v_{l}\right)\right)\right\rangle$, and $\widetilde{p}^{-}=\left\langle\left[\min _{l}\left(s_{\theta_{l}}\right), \min _{l}\left(s_{\tau_{l}}\right)\right]\right.$, $\left.\left(\min _{l}\left(u_{l}\right), \max _{l}\left(v_{l}\right)\right)\right\rangle$; then

$$
\widetilde{p}^{-} \leq \operatorname{PFULPWAA}\left(\widetilde{p}_{1}, \widetilde{p}_{2}, \ldots, \widetilde{p}_{n}\right) \leq \widetilde{p}^{+}
$$

Proof. (1) According to Definition 11 and formula (13) in Theorem 5, we obtain

$$
\begin{aligned}
& \operatorname{PFULPWAA}\left(\widetilde{p}_{1}, \widetilde{p}_{2}, \ldots, \widetilde{p}_{n}\right) \\
& =\left(\frac{T_{1}}{\sum_{l=1}^{n} T_{l}} \widetilde{p}_{1}\right) \oplus\left(\frac{T_{2}}{\sum_{l=1}^{n} T_{l}} \widetilde{p}_{2}\right) \oplus \cdots \\
& \quad \oplus\left(\frac{T_{n}}{\sum_{l=1}^{n} T_{l}} \widetilde{p}_{n}\right) \\
& =\left(\frac{T_{1}}{\sum_{l=1}^{n} T_{l}} \tilde{p}\right) \oplus\left(\frac{T_{2}}{\sum_{l=1}^{n} T_{l}} \widetilde{p}\right) \oplus \cdots \\
& \quad\left(\frac{T_{n}}{\sum_{l=1}^{n} T_{l}} \widetilde{p}\right)=\left(\frac{\sum_{l=1}^{n} T_{l}}{\sum_{l=1}^{n} T_{l}} \widetilde{p}\right)=\widetilde{p} .
\end{aligned}
$$

(2) Since $\min _{l}\left(s_{\theta_{l}}\right) \leq s_{\theta_{l}} \leq \max _{l}\left(s_{\theta_{l}}\right), \min _{l}\left(s_{\tau_{l}}\right) \leq s_{\tau_{l}} \leq$ $\max _{l}\left(s_{\tau_{l}}\right), \min _{l}\left(u_{l}\right) \leq u_{l} \leq \max _{l}\left(u_{l}\right)$, and $\min _{l}\left(v_{l}\right) \leq v_{l} \leq$ $\max _{l}\left(v_{l}\right)$, then we obtain

$$
\begin{aligned}
& \sum_{l=1}^{n} \frac{T_{l}}{\sum_{l=1}^{n} T_{l}} \theta_{l} \leq \sum_{l=1}^{n} \frac{T_{l}}{\sum_{l=1}^{n} T_{l}} \max _{l}\left(\theta_{l}\right), \\
& \sum_{l=1}^{n} \frac{T_{l}}{\sum_{l=1}^{n} T_{l}} \tau_{l} \leq \sum_{l=1}^{n} \frac{T_{l}}{\sum_{l=1}^{n} T_{l}} \max _{l}\left(\tau_{l}\right), \\
& \sqrt{1-\prod_{l=1}^{n}\left(1-u_{l}^{2}\right)^{T_{l} / \sum_{l=1}^{n} T_{l}}} \\
& \leq \sqrt{1-\prod_{l=1}^{n}\left(1-\left(\max _{l}\left(u_{l}\right)\right)^{2}\right)^{T_{l} / \sum_{l=1}^{n} T_{l}}}, \\
& \prod_{l=1}^{n}\left(\min _{l}\left(v_{l}\right)\right)^{T_{l} / \sum_{l=1}^{n} T_{l}} \leq \prod_{l=1}^{n} v_{l}^{T_{l} / \sum_{l=1}^{n} T_{l}} .
\end{aligned}
$$

Furthermore, according to Definition 6, we obtain

$$
\begin{aligned}
& S\left(\operatorname{PFULPWAA}\left(\widetilde{p}_{1}, \widetilde{p}_{2}, \ldots, \widetilde{p}_{n}\right)\right) \\
& \quad \leq S\left(\operatorname{PFULPWAA}\left(\tilde{p}^{+}, \tilde{p}^{+}, \ldots, \widetilde{p}^{+}\right)\right) .
\end{aligned}
$$

Thus, we should analyze the following two situations.

(1) If $S\left(\operatorname{PFULPWAA}\left(\widetilde{p}_{1}, \widetilde{p}_{2}, \ldots, \widetilde{p}_{n}\right)\right)$ $S\left(\operatorname{PFULPWAA}\left(\widetilde{p}^{+}, \widetilde{p}^{+}, \ldots, \widetilde{p}^{+}\right)\right)$, according to Definition 8 and the idempotency of the PFULPWAA operator, we obtain

$$
\begin{aligned}
& \operatorname{PFULPWAA}\left(\tilde{p}_{1}, \widetilde{p}_{2}, \ldots, \tilde{p}_{n}\right) \\
& \quad<\operatorname{PFULPWAA}\left(\widetilde{p}^{+}, \tilde{p}^{+}, \ldots, \widetilde{p}^{+}\right)=\widetilde{p}^{+} .
\end{aligned}
$$


(2) If $S\left(\operatorname{PFULPWAA}\left(\widetilde{p}_{1}, \widetilde{p}_{2}, \ldots, \widetilde{p}_{n}\right)\right)$ $S\left(\operatorname{PFULPWAA}\left(\widetilde{p}^{+}, \tilde{p}^{+}, \ldots, \tilde{p}^{+}\right)\right)$, according to formulas (31), we obtain

$$
\begin{aligned}
& \sum_{l=1}^{n} \frac{T_{l}}{\sum_{l=1}^{n} T_{l}} \theta_{l}=\sum_{l=1}^{n} \frac{T_{l}}{\sum_{l=1}^{n} T_{l}} \max _{l}\left(\theta_{l}\right), \\
& \sum_{l=1}^{n} \frac{T_{l}}{\sum_{l=1}^{n} T_{l}} \tau_{l}=\sum_{l=1}^{n} \frac{T_{l}}{\sum_{l=1}^{n} T_{l}} \max _{l}\left(\tau_{l}\right), \\
& \sqrt{1-\prod_{l=1}^{n}\left(1-u_{l}^{2}\right)^{T_{l} / \sum_{l=1}^{n} T_{l}}} \\
& =\sqrt{1-\prod_{l=1}^{n}\left(1-\left(\max _{l}\left(u_{l}\right)\right)^{2}\right)^{T_{l} / \sum_{l=1}^{n} T_{l}}}, \\
& \prod_{l=1}^{n}\left(\min _{l}\left(v_{l}\right)\right)^{T_{l} / \sum_{l=1}^{n} T_{l}}=\prod_{l=1}^{n} v_{l}^{T_{l} / \sum_{l=1}^{n} T_{l}}
\end{aligned}
$$

and according to Definition 7, we obtain

$$
\begin{aligned}
& A\left(\operatorname{PFUlpWAA}\left(\widetilde{p}_{1}, \widetilde{p}_{2}, \ldots, \widetilde{p}_{n}\right)\right) \\
& \quad=A\left(\operatorname{PFUlPWAA}\left(\widetilde{p}^{+}, \widetilde{p}^{+}, \ldots, \widetilde{p}^{+}\right)\right) ;
\end{aligned}
$$

then according to Definition 8 and the idempotency of the PFULPWAA operator, we obtain

$$
\begin{aligned}
& \operatorname{PFULPWAA}\left(\widetilde{p}_{1}, \widetilde{p}_{2}, \ldots, \widetilde{p}_{n}\right) \\
& \quad=\operatorname{PFULPWAA}\left(\widetilde{p}^{+}, \widetilde{p}^{+}, \ldots, \widetilde{p}^{+}\right)=\widetilde{p}^{+} .
\end{aligned}
$$

Therefore, we can obtain PFULPWAA $\left(\widetilde{p}_{1}, \widetilde{p}_{2}, \ldots, \widetilde{p}_{n}\right) \leq \tilde{p}^{+}$.

Similarly, we have $\widetilde{p}^{-}=\operatorname{PFULPWAA}\left(\widetilde{p}^{-}, \tilde{p}^{-}, \ldots, \widetilde{p}^{-}\right) \leq$ $\operatorname{PFULPWAA}\left(\widetilde{p}_{1}, \widetilde{p}_{2}, \ldots, \widetilde{p}_{n}\right)$. $\tilde{p}^{+}$.

Therefore, we obtain $\widetilde{p}^{-} \leq \operatorname{PFULPWAA}\left(\widetilde{p}_{1}, \widetilde{p}_{2}, \ldots, \widetilde{p}_{n}\right) \leq$

4.2. The PFULPWGA Operator. Based on PFULPWAA operator and geometric mean, the PFULPWGA operator is defined as follows.

Definition 14. Suppose $\widetilde{P}$ is the set of all PFULVs, $\widetilde{p}_{l}=$ $\left\langle\left[s_{\theta_{l}}, s_{\tau_{l}}\right],\left(u_{l}, v_{l}\right)\right\rangle(l=1,2, \ldots, n)$ is a collection of PFULVs, and PFULPWGA: $\widetilde{P}^{n} \rightarrow \widetilde{P}$, and if

$$
\operatorname{PFULPWGA}\left(\widetilde{p}_{1}, \tilde{p}_{2}, \ldots, \widetilde{p}_{n}\right)=\bigotimes_{l=1}^{n} \tilde{p}_{l}^{T_{l} / \sum_{l=1}^{n} T_{l}},
$$

where $T_{l}=\prod_{j=1}^{l-1} S\left(\widetilde{p}_{j}\right)(l=2,3, \ldots, n), T_{1}=1$, and $S\left(\widetilde{p}_{j}\right)$ is the score value of a PFULV $\tilde{p}_{j}$, then PFULPWGA is called the Pythagorean fuzzy uncertain linguistic prioritized weighted geometric aggregation operator.
Theorem 15. Suppose that $\widetilde{p}_{l}=\left\langle\left[s_{\theta_{l}}, s_{\tau_{l}}\right],\left(u_{l}, v_{l}\right)\right\rangle(l=1,2$, $\ldots, n)$ is a collection of PFULVs; then the aggregating value by PFULPWGA operator is still a PFULV and

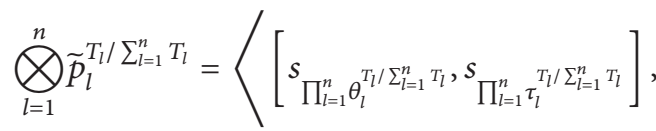

$$
\begin{aligned}
& \left.\left(\prod_{l=1}^{n} u_{l}^{T_{l} / \sum_{l=1}^{n} T_{l}}, \sqrt{1-\prod_{l=1}^{n}\left(1-v_{l}^{2}\right)^{T_{l} / \sum_{l=1}^{n} T_{l}}}\right)\right\rangle,
\end{aligned}
$$

where $T_{l}=\prod_{j=1}^{l-1} S\left(\widetilde{p}_{j}\right)(l=2,3, \ldots, n), T_{1}=1$, and $S\left(\widetilde{p}_{j}\right)$ is the score value of the $\widetilde{p}_{l}$.

Proof. Similar to Theorem 12, the proof of Theorem 15 is omitted.

Obviously, the PFULPWGA operator also has idempotency and boundness, and the proofs are omitted here.

\section{Pythagorean Fuzzy Uncertain Linguistic Maclaurin Symmetric Mean Aggregation Operators}

In this section, we will extend the MSM to PFULS and propose the PFULMSMA and WPFULMSMA operators.

\subsection{The PFULMSMA Operator}

Definition 16. Suppose $\widetilde{P}$ is the set of all PFULVs, $\widetilde{p}_{l}=$ $\left\langle\left[s_{\theta_{l}}, s_{\tau_{l}}\right],\left(u_{l}, v_{l}\right)\right\rangle(l=1,2, \ldots, n)$ is a collection of PFULVs, $\kappa=1,2, \ldots, n$, and PFULMSMA: $\widetilde{P}^{n} \rightarrow \widetilde{P}$, and if

$$
\begin{aligned}
& \operatorname{PFULMSMA~}^{(\kappa)}\left(\tilde{p}_{1}, \tilde{p}_{2}, \ldots, \widetilde{p}_{n}\right) \\
& =\left(\frac{\bigoplus_{1 \leq l_{1}<\cdots<l_{\kappa} \leq n}\left(\otimes_{j=1}^{\kappa} \tilde{p}_{l_{j}}\right)}{C_{n}^{\kappa}}\right)^{1 / \kappa},
\end{aligned}
$$

where $C_{n}^{\kappa}$ is the binomial coefficient and $\left\{l_{1}, l_{2}, \ldots, l_{\kappa}\right\}$ traverses all the $k$-tuple combinations of $\{1,2, \ldots, n\}$, then PFULMSMA is called the Pythagorean fuzzy uncertain linguistic Maclaurin symmetric mean aggregation operator.

Theorem 17. Suppose $\widetilde{p}_{l}=\left\langle\left[s_{\theta_{l}}, s_{\tau_{l}}\right],\left(u_{l}, v_{l}\right)\right\rangle(l=1,2, \ldots, n)$ is a collection of PFULVs, and $\kappa=1,2, \ldots, n$; then the aggregating value by PFULMSMA operator is still a PFULV, and

$$
\begin{aligned}
& \left(\frac{\bigoplus_{1 \leq l_{1}<\cdots<l_{1} \leq n} \otimes_{j=1}^{\kappa} \tilde{P}_{l_{j}}}{C_{n}^{\kappa}}\right)^{1 / \kappa}=\left\langle\left[ s_{\left(\Sigma_{1 \leq 1_{1}<\cdots<l_{k} \leq n} \Pi_{j=1}^{\kappa} \theta_{j} / C_{n}^{k}\right)^{1 / \kappa}},\right.\right. \\
& \left.s_{\left(\sum_{1 \leq 1_{1}<\cdots l_{k} \leq n} \prod_{j=1}^{\kappa} \tau_{j_{j}} / C_{n}^{\kappa}\right)^{1 / \kappa}}\right], \\
& \left(\sqrt{\left(1-\left(\prod_{1 \leq l_{1}<\cdots<l_{k} \leq n}\left(1-\prod_{j=1}^{\kappa} u_{l_{j}}^{2}\right)\right)^{1 / C_{n}^{\kappa}}\right)^{1 / \kappa}},\right. \\
& \left.\sqrt{\left.1-\left(1-\left(\prod_{1 \leq l_{1}<\cdots<l_{\kappa} \leq n}\left(1-\prod_{j=1}^{\kappa}\left(1-v_{l_{j}}^{2}\right)\right)\right)^{1 / C_{n}^{\kappa}}\right)^{1 / \kappa}\right)}\right\rangle .
\end{aligned}
$$


Proof. According to the operational laws of PFULVs, we have

$$
\begin{aligned}
& \bigotimes_{j=1}^{\kappa} \widetilde{p}_{l_{j}}=\left\langle\left[s_{\prod_{j=1}^{\kappa} \theta_{l_{j}}}, s_{\prod_{j=1}^{\kappa} \tau_{l_{j}}}\right],\left(\prod_{j=1}^{\kappa} u_{l_{j}},\right.\right. \\
& \left.\left.\sqrt{1-\prod_{j=1}^{\kappa}\left(1-v_{l_{j}}^{2}\right)}\right)\right\rangle \\
& \bigoplus_{1 \leq l_{1}<\cdots<l_{\kappa} \leq n} \bigotimes_{j=1}^{\kappa} \widetilde{p}_{l_{j}}=\left\langle\left[ s_{\sum_{1 \leq l_{1}<\cdots<l_{\kappa} \leq n} \prod_{j=1}^{\kappa} \theta_{l_{j}}},\right.\right. \\
& \left.s_{\sum_{1 \leq l_{1}<\ldots<l_{\kappa} \leq n} \prod_{j=1}^{\kappa} \tau_{l_{j}}}\right], \\
& \left(\sqrt{1-\prod_{1 \leq l_{1}<\cdots<l_{\kappa} \leq n}\left(1-\prod_{j=1}^{\kappa} u_{l_{j}}^{2}\right)},\right. \\
& \left.\left.\prod_{1 \leq l_{1}<\cdots<l_{\kappa} \leq n} \sqrt{1-\prod_{j=1}^{\kappa}\left(1-v_{l_{j}}^{2}\right)}\right)\right\rangle ;
\end{aligned}
$$

then

$$
\begin{aligned}
& \frac{\bigoplus_{1 \leq l_{1}<\cdots<l_{\kappa} \leq n} \otimes_{j=1}^{\kappa} \tilde{p}_{l_{j}}}{C_{n}^{\kappa}}=\left\langle\left[ s_{\left(\sum_{1 \leq l_{1}<\cdots<l_{\kappa} \leq n} \prod_{j=1}^{\kappa} \theta_{i_{j}}\right) / C_{n}^{\kappa},}\right.\right. \\
& (s_{\left.\left(\sum_{1 \leq l_{1}<\cdots<l_{\kappa} \leq n} \prod_{j=1}^{\kappa} \tau_{l_{j}}\right) / C_{n}^{\kappa}\right]} \underbrace{}_{1-\left(\prod_{1 \leq l_{1}<\cdots<l_{\kappa} \leq n}\left(1-\prod_{j=1}^{\kappa} u_{l_{j}}^{2}\right)\right)^{1 / C_{n}^{\kappa}}}, \\
& \left.\left(\prod_{1 \leq l_{1}<\cdots<l_{\kappa} \leq n} \sqrt{\left.1-\prod_{j=1}^{\kappa}\left(1-v_{l_{j}}^{2}\right)\right)^{1 / C_{n}^{\kappa}}}\right)\right\rangle ;
\end{aligned}
$$

therefore

$$
\begin{aligned}
& \left(\frac{\bigoplus_{1 \leq l_{1}<\cdots<l_{\kappa} \leq n} \otimes_{j=1}^{\kappa} \tilde{p}_{l_{j}}}{C_{n}^{\kappa}}\right)^{1 / \kappa}=\left\langle\left[ s_{\left(\left(\sum_{1 \leq l_{1}<\cdots<l_{k} \leq n} \prod_{j=1}^{\kappa} \theta_{l_{j}}\right) / C_{n}^{\kappa}\right)^{1 / \kappa},}\right.\right. \\
& s_{\left.\left(\left(\sum_{1 \leq l_{1}<\cdots<l_{k} \leq n} \prod_{j=1}^{\kappa} \tau_{l_{j}}\right) / C_{n}^{\kappa}\right)^{1 / \kappa}\right]} \\
& \left(\sqrt{\left(1-\left(\prod_{1 \leq l_{1}<\cdots<l_{\kappa} \leq n}\left(1-\prod_{j=1}^{\kappa} u_{l_{j}}^{2}\right)\right)^{1 / C_{n}^{\kappa}}\right)^{1 / \kappa}},\right. \\
& \left.\left.\sqrt{1-\left(1-\left(\prod_{1 \leq l_{1}<\cdots<l_{\kappa} \leq n}\left(1-\prod_{j=1}^{\kappa}\left(1-v_{l_{j}}^{2}\right)\right)\right)^{1 / C_{n}^{\kappa}}\right)^{1 / \kappa}}\right)\right\rangle ;
\end{aligned}
$$

that is, formula (40) is right.
In addition, since

$$
\begin{aligned}
& 0 \leq\left(\frac{\sum_{1 \leq l_{1}<\cdots<l_{\kappa} \leq n} \prod_{j=1}^{\kappa} \theta_{l_{j}}}{C_{n}^{\kappa}}\right)^{1 / \kappa} \leq t-1, \\
& 0 \leq\left(\frac{\sum_{1 \leq l_{1}<\cdots<l_{\kappa} \leq n} \prod_{j=1}^{\kappa} \tau_{l_{j}}}{C_{n}^{\kappa}}\right)^{1 / \kappa} \leq t-1, \\
& \left(\frac{\sum_{1 \leq l_{1}<\cdots<l_{\kappa} \leq n} \prod_{j=1}^{\kappa} \theta_{l_{j}}}{C_{n}^{\kappa}}\right) \leq\left(\frac{\sum_{1 \leq l_{1}<\cdots<l_{\kappa} \leq n} \prod_{j=1}^{\kappa} \tau_{l_{j}}}{C_{n}^{\kappa}}\right), \\
& 0 \leq \sqrt{\left(1-\left(\prod_{1 \leq l_{1}<\cdots<l_{\kappa} \leq n}\left(1-\prod_{j=1}^{\kappa} u_{l_{j}}^{2}\right)\right)^{1 / C_{n}^{\kappa}}\right)^{1 / \kappa}} \leq 1, \\
& \left.0 \sqrt{1-\left(1-\left(\prod_{1 \leq l_{1}<\cdots<l_{\kappa} \leq n}\left(1-\prod_{j=1}^{\kappa}\left(1-v_{l_{j}}^{2}\right)\right)^{1 / C_{n}^{\kappa}}\right.\right.}\right)^{1 / \kappa}
\end{aligned}
$$

$\leq 1$,

0

$$
\begin{aligned}
\leq & \left(1-\left(\prod_{1 \leq l_{1}<\cdots<l_{\kappa} \leq n}\left(1-\prod_{j=1}^{\kappa} u_{l_{j}}^{2}\right)\right)^{1 / C_{n}^{\kappa}}\right)^{1 / \kappa}+1 \\
& -\left(1-\left(\prod_{1 \leq l_{1}<\cdots<l_{\kappa} \leq n}\left(1-\prod_{j=1}^{\kappa}\left(1-v_{l_{j}}^{2}\right)\right)\right)^{1 / C_{n}^{\kappa}}\right)^{1 / \kappa} \\
\leq & \left(1-\left(\prod_{1 \leq l_{1}<\cdots<l_{\kappa} \leq n}\left(1-\prod_{j=1}^{\kappa} u_{l_{j}}^{2}\right)\right)^{1 / C_{n}^{\kappa}}\right)^{1 / \kappa}+1 \\
& -\left(1-\left(\prod_{1 \leq l_{1}<\cdots<l_{\kappa} \leq n}\left(1-\prod_{j=1}^{\kappa}\left(u_{l_{j}}^{2}\right)\right)\right)^{1 / C_{n}^{\kappa}}\right)^{1 / \kappa}=1
\end{aligned}
$$

formula (40) is still a PFULV, and the proof of Theorem 17 is finished.

In what follows, some properties of the PFULMSMA operator will be explored.

Theorem 18. Suppose that $\widetilde{p}_{l}=\left\langle\left[s_{\theta_{l}}, s_{\tau_{l}}\right],\left(u_{l}, v_{l}\right)\right\rangle$ and $\tilde{q}_{i}=$ $\left\langle\left[s_{\theta_{l}^{\prime}}, s_{\tau_{l}^{\prime}}\right],\left(u_{l}^{\prime}, v_{l}^{\prime}\right)\right\rangle(l=1,2, \ldots, n)$ are two collections of PFULVs, and $\kappa=1,2, \ldots, n$, and then the PFULMSMA operator has the following properties:

(1) (Idempotency). Suppose all $\tilde{p}_{l}=\left\langle\left[s_{\theta_{l}}, s_{\tau_{l}}\right],\left(u_{l}, v_{l}\right)\right\rangle(l=$ $1,2, \ldots, n)$ are equal; that is, $\widetilde{p}_{l}=\widetilde{p}=\left\langle\left[s_{\theta}, s_{\tau}\right]\right.$, $(u, v)\rangle(l=1,2, \ldots, n)$, and then

$$
\operatorname{PFULMSMA}{ }^{(\kappa)}\left(\widetilde{p}_{1}, \widetilde{p}_{2}, \ldots, \widetilde{p}_{n}\right)=\tilde{p}
$$


(2) (Commutativity). Suppose $\tilde{p}_{l}$ is any permutation of $\tilde{q}_{i}(l=1,2, \ldots, n) ;$ then

$$
\begin{aligned}
& \operatorname{PFULMSMA}^{(\kappa)}\left(\widetilde{p}_{1}, \tilde{p}_{2}, \ldots, \widetilde{p}_{n}\right) \\
& \quad=\operatorname{PFULMSMA}{ }^{(\kappa)}\left(\widetilde{q}_{1}, \tilde{q}_{2}, \ldots, \widetilde{q}_{n}\right) .
\end{aligned}
$$

(3) (Monotonicity). Suppose $\widetilde{p}_{i} \geq \widetilde{q}_{i}(i=1,2, \ldots, n)$; that is, $s_{\theta_{l}} \geq s_{\theta_{l}^{\prime}}, s_{\tau_{l}} \geq s_{\tau_{l}^{\prime}}, u_{l} \geq u_{l}^{\prime}, v_{l} \geq v_{l}^{\prime}$ for all $l=$ $1,2, \ldots, n$, and then

$$
\begin{aligned}
& \operatorname{PFULMSMA}^{(\kappa)}\left(\widetilde{p}_{1}, \tilde{p}_{2}, \ldots, \widetilde{p}_{n}\right) \\
& \quad \geq \operatorname{PFULMSMA}{ }^{(\kappa)}\left(\widetilde{q}_{1}, \tilde{q}_{2}, \ldots, \widetilde{q}_{n}\right) .
\end{aligned}
$$

(4) (Boundness). Suppose $\widetilde{p}^{+}=\left\langle\left[\max _{l}\left(s_{\theta_{l}}\right), \max _{l}\left(s_{\tau_{l}}\right)\right]\right.$, $\left.\left(\max _{l}\left(u_{l}\right), \min _{l}\left(v_{l}\right)\right)\right\rangle$ and $\widetilde{p}^{-}=\left\langle\left[\min _{l}\left(s_{\theta_{l}}\right), \min _{l}\left(s_{\tau_{l}}\right)\right]\right.$, $\left.\left(\min _{l}\left(u_{l}\right), \max _{l}\left(v_{l}\right)\right)\right\rangle$; then

$$
\widetilde{p}^{-} \leq \operatorname{PFULMSMA}{ }^{(\kappa)}\left(\tilde{p}_{1}, \tilde{p}_{2}, \ldots, \widetilde{p}_{n}\right) \leq \tilde{p}^{+}
$$

Proof. (1) Since $\widetilde{p}_{l}=\widetilde{p}=\left\langle\left[s_{\theta}, s_{\tau}\right],(u, v)\right\rangle(l=1,2, \ldots, n)$, according to Theorem 17, we obtain

$$
\begin{aligned}
& \left(\frac{\bigoplus_{1 \leq l_{1}<\cdots<l_{\kappa} \leq n} \otimes_{j=1}^{\kappa} \tilde{p}_{l_{j}}}{C_{n}^{\kappa}}\right)^{1 / \kappa}=\left\langle\left[ s_{\left(\left(\sum_{1 \leq 1_{1}<\cdots l_{k} \leq n} \prod_{j=1}^{\kappa} \theta_{l_{j}}\right) / C_{n}^{\kappa}\right)^{1 / \kappa},},\right.\right. \\
& s_{\left.\left(\left(\sum_{1 \leq 1_{1}<\cdots l_{k} \leq n} \prod_{j=1}^{\kappa} \tau_{l_{j}}\right) / C_{n}^{\kappa}\right)^{1 / \kappa}\right],}, \\
& \left(\sqrt{\left(1-\left(\prod_{1 \leq l_{1}<\cdots<l_{k} \leq n}\left(1-\prod_{j=1}^{\kappa} u_{l_{j}}^{2}\right)\right)^{1 / C_{n}^{\kappa}}\right)^{1 / \kappa}},\right. \\
& \left.\left.\sqrt{1-\left(1-\left(\prod_{1 \leq l_{1}<\cdots<l_{\kappa} \leq n}\left(1-\prod_{j=1}^{\kappa}\left(1-v_{l_{j}}^{2}\right)\right)\right)^{1 / C_{n}^{\kappa}}\right)^{1 / \kappa}}\right)\right\rangle \\
& =\left\langle\left[s_{\left(\left(\sum_{1 \leq 1_{1}<\cdots<l_{k} \leq n} \theta^{\kappa}\right) / C_{n}^{\kappa}\right)^{1 / \kappa},} s_{\left(\left(\sum_{1 \leq 1_{1}<\cdots<l_{k} \leq n} \tau^{\kappa}\right) / C_{n}^{\kappa}\right)^{1 / \kappa}}\right],\right. \\
& \left(\sqrt{\left(1-\left(\prod_{1 \leq l_{1}<\ldots<l_{\kappa} \leq n}\left(1-u^{2 \kappa}\right)\right)^{1 / C_{n}^{\kappa}}\right)^{1 / \kappa}}\right. \\
& \left.\left.\sqrt{1-\left(1-\left(\prod_{1 \leq l_{1}<\ldots<l_{\kappa} \leq n}\left(1-\left(1-v^{2}\right)^{\kappa}\right)\right)^{1 / C_{n}^{\kappa}}\right)^{1 / \kappa}}\right)\right\rangle \\
& =\left\langle\left[s_{\left(C_{n}^{\kappa} \theta^{\kappa} / C_{n}^{K}\right)^{1 / \kappa},} s_{\left(C_{n}^{\kappa} \tau^{\kappa} / C_{n}^{K}\right)^{1 / \kappa}}\right],\right.
\end{aligned}
$$

$\left(\sqrt{\left(1-\left(\left(1-u^{2 \kappa}\right)^{C_{n}^{\kappa}}\right)^{1 / C_{n}^{\kappa}}\right)^{1 / \kappa}}\right.$,

$\left.\left.\sqrt{1-\left(1-\left(\left(1-\left(1-v^{2}\right)^{\kappa}\right)^{C_{n}^{\kappa}}\right)^{1 / C_{n}^{\kappa}}\right)^{1 / \kappa}}\right)\right\rangle=\left\langle\left[s_{\theta}, s_{\tau}\right],(u\right.$,

$v)\rangle$.

(2) Since $\widetilde{q}_{i}$ is any permutation of $\widetilde{p}_{l}(l=1,2, \ldots, n)$ according to Theorem 17 , we can easily obtain this conclusion.

(3) Since $s_{\theta_{l}} \geq s_{\theta_{l}^{\prime}}, s_{\tau_{l}} \geq s_{\tau_{l}^{\prime}}, u_{l} \geq u_{l}^{\prime}$, and $v_{l} \geq v_{l}^{\prime}$ for all $l=1,2, \ldots, n$, then we have

$$
\begin{aligned}
& \prod_{j=1}^{\kappa} \theta_{l_{j}} \geq \prod_{j=1}^{\kappa} \theta_{l_{j}}^{\prime}, \\
& \prod_{j=1}^{\kappa} \tau_{l_{j}} \geq \prod_{j=1}^{\kappa} \tau_{l_{j}}^{\prime}, \\
& \prod_{j=1}^{\kappa} u_{l_{j}}^{2} \geq \prod_{j=1}^{\kappa} u_{l_{j}}^{\prime 2},
\end{aligned}
$$$$
\prod_{j=1}^{\kappa}\left(1-v_{l_{j}}^{2}\right) \geq \prod_{j=1}^{\kappa}\left(1-v_{l_{j}}^{\prime 2}\right)
$$$$
\sum_{1 \leq l_{1}<\cdots<l_{\kappa} \leq n} \prod_{j=1}^{\kappa} \theta_{l_{j}} \geq \sum_{1 \leq l_{1}<\cdots<l_{\kappa} \leq n} \prod_{j=1}^{\kappa} \theta_{l_{j}}^{\prime}
$$$$
\sum_{1 \leq l_{1}<\cdots<l_{\kappa} \leq n} \prod_{j=1}^{\kappa} \tau_{l_{j}} \geq \sum_{1 \leq l_{1}<\cdots<l_{\kappa} \leq n} \prod_{j=1}^{\kappa} \tau_{l_{j}}^{\prime},
$$$$
\prod_{1 \leq l_{1}<\cdots<l_{\kappa} \leq n}\left(1-\prod_{j=1}^{\kappa} u_{l_{j}}^{2}\right) \leq \prod_{1 \leq l_{1}<\cdots<l_{\kappa} \leq n}\left(1-\prod_{j=1}^{\kappa} u_{l_{j}}^{\prime 2}\right),
$$$$
\prod_{1 \leq l_{1}<\cdots<l_{\kappa} \leq n}\left(1-\prod_{j=1}^{\kappa}\left(1-v_{l_{j}}^{2}\right)\right)
$$$$
\leq \prod_{1 \leq l_{1}<\cdots<l_{\kappa} \leq n}\left(1-\prod_{j=1}^{\kappa}\left(1-v_{l_{j}}^{\prime 2}\right)\right)
$$

and then

$$
\begin{aligned}
& \left(\frac{\sum_{1 \leq l_{1}<\cdots<l_{\kappa} \leq n} \prod_{j=1}^{\kappa} \theta_{l_{j}}}{C_{n}^{\kappa}}\right)^{1 / \kappa} \geq\left(\frac{\sum_{1 \leq l_{1}<\cdots<l_{\kappa} \leq n} \prod_{j=1}^{\kappa} \theta_{l_{j}}^{\prime}}{C_{n}^{\kappa}}\right)^{1 / \kappa}, \\
& \left(\frac{\sum_{1 \leq l_{1}<\cdots<l_{\kappa} \leq n} \prod_{j=1}^{\kappa} \tau_{l_{j}}}{C_{n}^{\kappa}}\right)^{1 / \kappa} \geq\left(\frac{\sum_{1 \leq l_{1}<\cdots<l_{\kappa} \leq n} \prod_{j=1}^{\kappa} \tau_{l_{j}}^{\prime}}{C_{n}^{\kappa}}\right)^{1 / \kappa},
\end{aligned}
$$




$$
\begin{aligned}
& \sqrt{\left(1-\left(\prod_{1 \leq l_{1}<\ldots<l_{\kappa} \leq n}\left(1-\prod_{j=1}^{\kappa} u_{l_{j}}^{2}\right)\right)^{1 / C_{n}^{\kappa}}\right)^{1 / \kappa}} \\
& \geq \sqrt{\left(1-\left(\prod_{1 \leq l_{1}<\ldots<l_{\kappa} \leq n}\left(1-\prod_{j=1}^{\kappa} u_{l_{j}}^{\prime 2}\right)\right)^{1 / C_{n}^{\kappa}}\right)^{1 / \kappa}}, \\
& \left.\sqrt{1-\left(1-\left(\prod_{1 \leq l_{1}<\cdots<l_{\kappa} \leq n}\left(1-\prod_{j=1}^{\kappa}\left(1-v_{l_{j}}^{2}\right)\right)\right)^{1 / C_{n}^{\kappa}}\right)^{1 / \kappa}}\right)^{1-\left(1-\left(\prod_{1 \leq l_{1}<\cdots<l_{\kappa} \leq n}\left(1-\prod_{j=1}^{\kappa}\left(1-v_{l_{j}}^{\prime 2}\right)\right)^{1 / C_{n}^{\kappa}}\right)^{1 / \kappa}\right.} .
\end{aligned}
$$

Furthermore, according to Definition 6, we obtain

$$
\begin{aligned}
& S\left(\operatorname{PFULMSMA}^{(\kappa)}\left(\widetilde{p}_{1}, \widetilde{p}_{2}, \ldots, \widetilde{p}_{n}\right)\right) \\
& \quad \geq S\left(\operatorname{PFULMSMA~}^{(\kappa)}\left(\widetilde{q}_{1}, \widetilde{q}_{2}, \ldots, \widetilde{q}_{n}\right)\right) .
\end{aligned}
$$

Thus, we should analyze the following two situations.

(a) If $S\left(\operatorname{PFULMSMA}^{(\kappa)}\left(\tilde{p}_{1}, \tilde{p}_{2}, \ldots, \tilde{p}_{n}\right)\right)>$ $S\left(\operatorname{PFULMSMA}^{(\kappa)}\left(\widetilde{q}_{1}, \widetilde{q}_{2}, \ldots, \widetilde{q}_{n}\right)\right)$, according to Definition 8 , we obtain

$$
\begin{aligned}
& \operatorname{PFULMSMA~}^{(\kappa)}\left(\tilde{p}_{1}, \widetilde{p}_{2}, \ldots, \tilde{p}_{n}\right) \\
& \quad \geq \operatorname{PFULMSMA~}^{(\kappa)}\left(\widetilde{q}_{1}, \widetilde{q}_{2}, \ldots, \widetilde{q}_{n}\right)
\end{aligned}
$$

(b) If $S\left(\operatorname{PFULMSMA}^{(\kappa)}\left(\widetilde{p}_{1}, \widetilde{p}_{2}, \ldots, \widetilde{p}_{n}\right)\right)=$ $S\left(\operatorname{PFULMSMA}^{(\kappa)}\left(\widetilde{q}_{1}, \tilde{q}_{2}, \ldots, \widetilde{q}_{n}\right)\right)$, and, according to formulas (51)-(54), we obtain

$$
\begin{aligned}
& \left(\frac{\sum_{1 \leq l_{1}<\cdots<l_{\kappa} \leq n} \prod_{j=1}^{\kappa} \theta_{l_{j}}}{C_{n}^{\kappa}}\right)^{1 / \kappa}=\left(\frac{\sum_{1 \leq l_{1}<\cdots<l_{\kappa} \leq n} \prod_{j=1}^{\kappa} \theta_{l_{j}}^{\prime}}{C_{n}^{\kappa}}\right)^{1 / \kappa}, \\
& \left(\frac{\sum_{1 \leq l_{1}<\cdots<l_{\kappa} \leq n} \prod_{j=1}^{\kappa} \tau_{l_{j}}}{C_{n}^{\kappa}}\right)^{1 / \kappa}=\left(\frac{\sum_{1 \leq l_{1}<\cdots<l_{\kappa} \leq n} \prod_{j=1}^{\kappa} \tau_{l_{j}}^{\prime}}{C_{n}^{\kappa}}\right)^{1 / \kappa}, \\
& \sqrt{\left(1-\left(\prod_{1 \leq l_{1}<\cdots<l_{\kappa} \leq n}\left(1-\prod_{j=1}^{\kappa} u_{l_{j}}^{2}\right)\right)^{1 / C_{n}^{\kappa}}\right)^{1 / \kappa}} \\
& =\sqrt{\left(1-\left(\prod_{1 \leq l_{1}<\cdots<l_{\kappa} \leq n}\left(1-\prod_{j=1}^{\kappa} u_{l_{j}}^{\prime 2}\right)\right)^{1 / C_{n}^{\kappa}}\right)^{1 / \kappa}}, \\
& \sqrt{1-\left(1-\left(\prod_{1 \leq l_{1}<\cdots<l_{\kappa} \leq n}\left(1-\prod_{j=1}^{\kappa}\left(1-v_{l_{j}}^{2}\right)\right)\right)^{1 / C_{n}^{\kappa}}\right)^{1 / \kappa}} \\
& =\sqrt{1-\left(1-\left(\prod_{1 \leq l_{1}<\cdots<l_{\kappa} \leq n}\left(1-\prod_{j=1}^{\kappa}\left(1-v_{l_{j}}^{\prime 2}\right)\right)\right)^{1 / C_{n}^{\kappa}}\right)^{1 / \kappa}} .
\end{aligned}
$$

and according to Definition 7, we obtain

$$
\begin{aligned}
& A\left(\operatorname{PFULMSMA}^{(\kappa)}\left(\widetilde{p}_{1}, \widetilde{p}_{2}, \ldots, \widetilde{p}_{n}\right)\right) \\
& \quad=A\left(\operatorname{PFULMSMA~}^{(\kappa)}\left(\widetilde{q}_{1}, \tilde{q}_{2}, \ldots, \tilde{q}_{n}\right)\right) ;
\end{aligned}
$$

then according to Definition 8, we obtain

$$
\begin{aligned}
& \operatorname{PFULMSMA~}^{(\kappa)}\left(\widetilde{p}_{1}, \widetilde{p}_{2}, \ldots, \tilde{p}_{n}\right) \\
& \quad=\operatorname{PFULMSMA~}^{(\kappa)}\left(\widetilde{q}_{1}, \widetilde{q}_{2}, \ldots, \widetilde{q}_{n}\right) .
\end{aligned}
$$

Therefore, we can obtain

$$
\begin{aligned}
& \operatorname{PFULMSMA}^{(\kappa)}\left(\widetilde{p}_{1}, \widetilde{p}_{2}, \ldots, \widetilde{p}_{n}\right) \\
& \quad \geq \operatorname{PFULMSMA~}^{(\kappa)}\left(\widetilde{q}_{1}, \widetilde{q}_{2}, \ldots, \widetilde{q}_{n}\right) .
\end{aligned}
$$

(4) According to the monotonicity of the PFULMSMA operator, we obtain

$$
\begin{aligned}
& \operatorname{PFULMSMA}^{(\kappa)}\left(\widetilde{q}^{-}, \tilde{q}^{-}, \ldots, \tilde{q}^{-}\right) \\
& \quad \leq \operatorname{PFULMSMA~}^{(\kappa)}\left(\widetilde{p}_{1}, \widetilde{p}_{2}, \ldots, \widetilde{p}_{n}\right) \\
& \quad \leq \operatorname{PFULMSMA~}^{(\kappa)}\left(\widetilde{q}^{+}, \tilde{q}^{+}, \ldots, \tilde{q}^{+}\right) .
\end{aligned}
$$

And according to the idempotency of the PFULMSMA operator, then we obtain

$$
\begin{aligned}
& \widetilde{q}^{-}=\operatorname{PFULMSMA}^{(\kappa)}\left(\widetilde{q}^{-}, \widetilde{q}^{-}, \ldots, \widetilde{q}^{-}\right) \\
& \widetilde{q}^{+}=\operatorname{PFULMSMA~}^{(\kappa)}\left(\widetilde{q}^{+}, \tilde{q}^{+}, \ldots, \tilde{q}^{+}\right) .
\end{aligned}
$$

Therefore,

$$
\widetilde{p}^{-} \leq \operatorname{PFULMSMA}^{(\kappa)}\left(\widetilde{p}_{1}, \widetilde{p}_{2}, \ldots, \widetilde{p}_{n}\right) \leq \widetilde{p}^{+}
$$

Now, the monotonicity of the PFULMSMA operator about the parameter $\kappa$ shall be analyzed. In the first place, we give two lemmas which shall be utilized in the following investigation.

Lemma 19 (see [38]). Suppose that $B=\left\{b_{1}, b_{2}, \ldots, b_{n}\right\}$ is a collection of nonnegative real numbers, and for $\kappa=1,2, \ldots, n$, then

$$
\begin{aligned}
& \operatorname{MSM}^{(1)}\left(b_{1}, b_{2}, \ldots, b_{n}\right) \geq \operatorname{MSM}^{(2)}\left(b_{1}, b_{2}, \ldots, b_{n}\right) \geq \cdots \\
& \quad \geq \operatorname{MSM}^{(n)}\left(b_{1}, b_{2}, \ldots, b_{n}\right)
\end{aligned}
$$

with equality iff $b_{1}=b_{2}=\cdots=b_{n}$. 
Lemma 20 (see [3]). Suppose that $p_{l}=\left\langle\left(u_{l}, v_{l}\right)\right\rangle(l=1,2$, $\ldots, n)$ is a collection of PFULVs, and $\kappa=1,2, \ldots, n$, and if

$$
\begin{aligned}
& h(\kappa)=\left(1-\left(\prod_{1 \leq l_{1}<\cdots<l_{\kappa} \leq n}\left(1-\prod_{j=1}^{\kappa} u_{l_{j}}\right)\right)^{1 / C_{n}^{\kappa}}\right)^{1 / \kappa}, \\
& g(\kappa)=1-(1 \\
& \left.-\left(\prod_{1 \leq l_{1}<\cdots<l_{\kappa} \leq n}\left(1-\prod_{j=1}^{\kappa}\left(1-v_{l_{j}}\right)\right)\right)^{1 / C_{n}^{\kappa}}\right)^{1 / \kappa}
\end{aligned}
$$

then $h(\kappa)$ and $g(\kappa)$ are monotonically decreasing and increasing about the parameter $\kappa$, respectively.

Theorem 21. Suppose that $\widetilde{p}_{l}=\left\langle\left[s_{\theta_{l}}, s_{\tau_{l}}\right],\left(u_{l}, v_{l}\right)\right\rangle(l=1,2$, $\ldots, n)$ is a collection of PFULVs, and $\kappa=1,2, \ldots, n$; then the PFULMSMA ${ }^{(\kappa)}\left(\widetilde{p}_{1}, \widetilde{p}_{2}, \ldots, \widetilde{p}_{n}\right)$ operator is monotonically decreasing about the parameter $\kappa$.

Proof. Suppose

$$
\begin{aligned}
& f_{1}(\kappa)=\left(\frac{\sum_{1 \leq l_{1}<\cdots<l_{\kappa} \leq n} \prod_{j=1}^{\kappa} \theta_{l_{j}}}{C_{n}^{\kappa}}\right)^{1 / \kappa}, \\
& f_{2}(\kappa)=\left(\frac{\sum_{1 \leq l_{1}<\cdots<l_{\kappa} \leq n} \prod_{j=1}^{\kappa} \tau_{l_{j}}}{C_{n}^{\kappa}}\right)^{1 / \kappa}, \\
& p^{\prime}(\kappa)=\sqrt{\left(1-\left(\prod_{1 \leq l_{1}<\cdots<l_{\kappa} \leq n}\left(1-\prod_{j=1}^{\kappa} u_{l_{j}}^{2}\right)\right)^{1 / C_{n}^{\kappa}}\right)^{1 / \kappa}}, \\
& q^{\prime}(\kappa) \\
& =\sqrt{1-\left(1-\left(\prod_{1 \leq l_{1}<\cdots<l_{\kappa} \leq n}\left(1-\prod_{j=1}^{\kappa}\left(1-v_{l_{j}}^{2}\right)\right)\right)^{1 / C_{n}^{\kappa}}\right)^{1 / \kappa}} .
\end{aligned}
$$

According to Lemma 19, we know that functions $f_{1}(\kappa)$ and $f_{2}(\kappa)$ are both monotonically decreasing about the parameter $\kappa$. In addition, according to formulas (65), we know that $p^{\prime}(\kappa)$ and $q^{\prime}(\kappa)$ are monotonically decreasing and increasing about the parameter $\kappa$, respectively.

Based on the score function in formula (40), we obtain

$$
\begin{aligned}
& S\left(\operatorname{PFULMSMA~}^{(\kappa)}\left(\tilde{p}_{1}, \tilde{p}_{2}, \ldots, \tilde{p}_{n}\right)\right) \\
& \quad=\frac{f_{1}(\kappa)+f_{2}(\kappa)}{4(t-1)}\left(p^{\prime 2}(\kappa)+1-q^{\prime 2}(\kappa)\right) .
\end{aligned}
$$

For any $\kappa \in[2, n]$ and $\kappa \in Z$, we obtain

$$
\begin{aligned}
& S\left(\operatorname{PFULMSMA~}^{(\kappa)}\left(\widetilde{p}_{1}, \widetilde{p}_{2}, \ldots, \widetilde{p}_{n}\right)\right) \\
& \quad-S\left(\operatorname{PFULMSMA~}^{(\kappa-1)}\left(\widetilde{p}_{1}, \widetilde{p}_{2}, \ldots, \widetilde{p}_{n}\right)\right)
\end{aligned}
$$

$$
\begin{aligned}
& =\frac{f_{1}(\kappa)+f_{2}(\kappa)}{4(t-1)}\left(p^{\prime 2}(\kappa)+1-q^{\prime 2}(\kappa)\right) \\
& -\frac{f_{1}(\kappa-1)+f_{2}(\kappa-1)}{4(t-1)}\left(p^{\prime 2}(\kappa-1)+1\right. \\
& \left.-q^{\prime 2}(\kappa-1)\right) \\
& <\frac{f_{1}(\kappa-1)+f_{2}(\kappa-1)}{4(t-1)}\left(p^{\prime 2}(\kappa-1)+1\right. \\
& \left.-q^{\prime 2}(\kappa-1)\right) \\
& -\frac{f_{1}(\kappa-1)+f_{2}(\kappa-1)}{4(t-1)}\left(p^{\prime 2}(\kappa-1)+1\right. \\
& \left.-q^{\prime 2}(\kappa-1)\right)=0 ;
\end{aligned}
$$

that is, $\quad S\left(\operatorname{PFULMSMA}^{(\kappa)}\left(\tilde{p}_{1}, \widetilde{p}_{2}, \ldots, \tilde{p}_{n}\right)\right)$

$S\left(\right.$ PFULMSMA $\left.^{(\kappa-1)}\left(\widetilde{p}_{1}, \widetilde{p}_{2}, \ldots, \widetilde{p}_{n}\right)\right)$ for all $\kappa$. Therefore, the PFULMSMA $^{(\kappa)}\left(\widetilde{p}_{1}, \widetilde{p}_{2}, \ldots, \widetilde{p}_{n}\right)$ operator is monotonically decreasing about the parameter $\kappa$.

5.2. The WPFULMSMA Operator. The WPFULMSMA operator can consider not only the interrelationship among the multi-input arguments, but also the importance of each aggregated element.

Definition 22. Suppose $\widetilde{P}$ is the set of all PFULVs, $\widetilde{p}_{l}=\left\langle\left[s_{\theta_{l}}\right.\right.$, $\left.\left.s_{\tau_{l}}\right],\left(u_{l}, v_{l}\right)\right\rangle(l=1,2, \ldots, n)$ is a collection of PFULVs, $\kappa=$ $1,2, \ldots, n, \omega=\left(\omega_{1}, \omega_{2}, \ldots, \omega_{n}\right)^{T}$ is the weight vector of $\widetilde{p}_{l}=$ $\left\langle\left[s_{\theta_{l}}, s_{\tau_{l}}\right],\left(u_{l}, v_{l}\right)\right\rangle(l=1,2, \ldots, n)$, and WPFULMSMA: $\widetilde{P}^{n} \rightarrow$ $\widetilde{P}$, and if

$$
\begin{aligned}
& \operatorname{WPFULMSMA}^{(\kappa)}\left(\tilde{p}_{1}, \widetilde{p}_{2}, \ldots, \tilde{p}_{n}\right) \\
& =\left(\frac{\bigoplus_{1 \leq l_{1}<\cdots<l_{\kappa} \leq n}\left(\bigotimes_{j=1}^{\kappa} \omega_{j} \tilde{p}_{l_{j}}\right)}{C_{n}^{\kappa}}\right)^{1 / \kappa},
\end{aligned}
$$

where $C_{n}^{\kappa}$ is the binomial coefficient and $\left\{l_{1}, l_{2}, \ldots, l_{\kappa}\right\}$ traverses all the $k$-tuple combinations of $\{1,2, \ldots, n\}$, then WPFULMSMA is called the weighted PFULMSMA operator.

Theorem 23. Suppose $\widetilde{p}_{l}=\left\langle\left[s_{\theta_{l}}, s_{\tau_{l}}\right],\left(u_{l}, v_{l}\right)\right\rangle(l=1,2, \ldots$, $n)$ is a collection of PFULVs, $\kappa=1,2, \ldots, n$, and $\omega=$ $\left(\omega_{1}, \omega_{2}, \ldots, \omega_{n}\right)^{T}$ is the weight vector of $\tilde{p}_{l}=\left\langle\left[s_{\theta_{l}}, s_{\tau_{l}}\right],\left(u_{l}\right.\right.$, $\left.\left.v_{l}\right)\right\rangle(l=1,2, \ldots, n)$, and then the aggregating value by WPFULMSMA operator is still a PFULV, and 


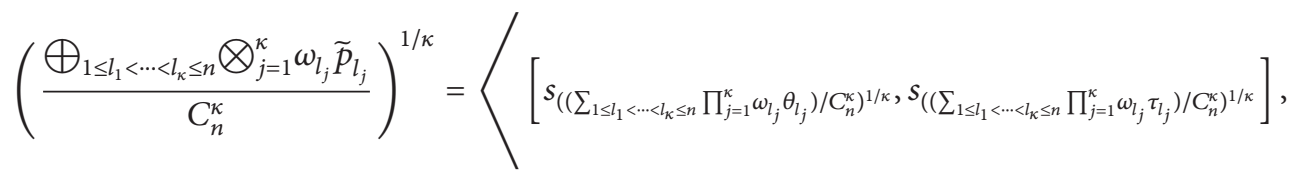

$$
\begin{aligned}
& \left(\sqrt{\left(1-\left(\prod_{1 \leq l_{1}<\ldots<l_{\kappa} \leq n}\left(1-\prod_{j=1}^{\kappa}\left(1-\left(1-u_{l_{j}}^{2}\right)^{\omega_{i_{j}}}\right)\right)\right)^{1 / C_{n}^{\kappa}}\right)^{1 / \kappa}},\right. \\
& \left.\left.\sqrt{1-\left(1-\left(\prod_{1 \leq l_{1}<\cdots<l_{\kappa} \leq n}\left(1-\prod_{j=1}^{\kappa}\left(1-v_{l_{j}}^{2 \omega_{l_{j}}}\right)\right)\right)^{1 / C_{n}^{\kappa}}\right)^{1 / \kappa}}\right)\right\rangle .
\end{aligned}
$$

Proof. Similar to Theorem 17, the proof of Theorem 23 is omitted.

Now, several special cases of the WPFULMSMA operator about the parameter $\kappa$ shall be discussed.

Case 1. When $\kappa=1$, formula (70) reduces to the following formula:

$$
\operatorname{WPFULMSMA}^{(1)}\left(\tilde{p}_{1}, p_{2}, \ldots, p_{n}\right)
$$

$$
\begin{aligned}
& =\left\langle\left[ s_{\left.(1 / n) \sum_{l=1}^{n} \omega_{l} \theta_{l}, s_{(1 / n) \sum_{l=1}^{n} \omega_{l} \tau_{l}}\right],}\right.\right. \\
& \left(\sqrt{\left.1-\left(\prod_{l=1}^{n}\left(1-u_{l}^{2}\right)^{\omega_{l}}\right)^{1 / n},\left(\prod_{l=1}^{n} v_{l}^{\omega_{l}}\right)^{1 / n}\right)}\right\rangle .
\end{aligned}
$$

Case 2. When $\kappa=2$, formula (70) reduces to a Pythagorean fuzzy uncertain linguistic weighted Bonferroni mean (PFUWBM) operator $(p=q=1)$.

$$
\begin{aligned}
& \text { WPFULMSMA }^{(2)}\left(\tilde{p}_{1}, p_{2}, \ldots, p_{n}\right)=\operatorname{PFULBM}^{(1,1)}\left(\tilde{p}_{1}, p_{2}, \ldots, p_{n}\right)=\left\langle s_{\left((1 / n(n-1)) \sum_{\substack{l, j=1 \\
l \neq j}}^{n} \omega_{l} \omega_{j} \theta_{l} \theta_{j}\right)^{1 / 2}}\right. \\
& \left.s_{\left((1 / n(n-1)) \sum_{\substack{l, j=1 \\
l \neq j}}^{n} \omega_{l} \omega_{j} \tau_{l} \tau_{j}\right)^{1 / 2}}\right],\left(\sqrt{\left(1-\left(\prod_{\substack{l, j=1 \\
l \neq j}}^{n}\left(1-\left(1-\left(1-u_{l}^{2}\right)^{\omega_{l}}\right)\left(1-\left(1-u_{j}^{2}\right)^{\omega_{j}}\right)\right)\right)^{1 / n(n-1)}\right)^{1 / 2}},\right. \\
& \left.\sqrt{1-\left(1-\left(\prod_{\substack{l, j=1 \\
l \neq j}}^{n}\left(1-\left(1-v_{l}^{2 \omega_{l}}\right)\left(1-v_{j}^{2 \omega_{j}}\right)\right)\right)^{1 / n(n-1)}\right)^{1 / 2}}\right\rangle .
\end{aligned}
$$

Case 3. When $\kappa=n$, formula (70) reduces to a Pythagorean fuzzy uncertain linguistic weighted Bonferroni mean (PFUWBM) operator $(p=1, q=0)$.

$$
\begin{aligned}
& \operatorname{WPFULMSMA}^{(n)}\left(\tilde{p}_{1}, \widetilde{p}_{2}, \ldots, \tilde{p}_{n}\right) \\
& \quad=\operatorname{PFULBM}^{(1,0)}\left(\tilde{p}_{1}, \tilde{p}_{2}, \ldots, \tilde{p}_{n}\right)=\left\langle\left[ s_{\left(\prod_{l=1}^{n} \omega_{l} \theta_{l}\right)^{1 / n},}\right.\right.
\end{aligned}
$$$$
\left.s_{\left(\prod_{l=1}^{n} \omega_{l} \tau_{l}\right)^{1 / n}}\right],\left(\sqrt{\left(\prod_{l=1}^{n}\left(1-\left(1-u_{l}^{2}\right)^{\omega_{l}}\right)\right)^{1 / n}}\right.
$$$$
\left.\left.\sqrt{1-\left(\prod_{l=1}^{n}\left(1-v_{l}^{2 \omega_{l}}\right)\right)^{1 / n}}\right)\right\rangle
$$ 
Next, the monotonicity of the WPFULMSMA operator about the parameter $\kappa$ shall be analyzed.

Theorem 24. Suppose that $\widetilde{p}_{l}=\left\langle\left[s_{\theta_{l}}, s_{\tau_{l}}\right],\left(u_{l}, v_{l}\right)\right\rangle(l=1,2$, $\ldots, n)$ is a collection of PFULVs, and $\kappa=1,2, \ldots, n$, and then the WPFULMSMA ${ }^{(\kappa)}\left(\widetilde{p}_{1}, \widetilde{p}_{2}, \ldots, \widetilde{p}_{n}\right)$ operator is monotonically decreasing about the parameter $\kappa$.

Proof. Similar to Theorem 21, the proof of Theorem 24 is omitted.

\section{A Group Decision-Making Method with Unknown Weight Based on Pythagorean Fuzzy Uncertain Linguistic Aggregation Operators}

In this section, we will develop a method to solve the MCGDM problems with unknown weight under Pythagorean fuzzy uncertain linguistic environment by utilizing the Pythagorean fuzzy uncertain linguistic aggregation operators and grey relational analysis (GRA) method.

For a MCGDM problem with PFULVs, suppose $A=$ $\left\{a_{1}, a_{2}, \ldots, a_{m}\right\}$ is the set of candidate alternatives and $C=$ $\left\{c_{1}, c_{2}, \ldots, c_{n}\right\}$ is the set of criteria, whose weight vector is completely unknown. Furthermore, suppose that $E=$ $\left\{e_{1}, e_{2}, \ldots, e_{q}\right\}$ is the set of experts, whose weight vector is also unknown but there is a prioritization relationship for experts $e_{1}>e_{2}>\cdots>e_{q}$. Assume that $R^{\delta}=\left[r_{i j}^{\delta}\right]_{m \times n}(\delta=$ $1,2, \ldots, q)$ is a decision matrix of MCGDM problem, where $r_{i j}^{\delta}=\left\langle\left[s_{\theta_{i j}}^{\delta}, s_{\tau_{i j}}^{\delta}\right],\left(u_{i j}^{\delta}, v_{i j}^{\delta}\right)\right\rangle(i=1,2, \ldots, m ; j=1,2, \ldots, n ; \delta=$ $1,2, \ldots, q)$ is the evaluation information expressed by the PFULV with respect to alternative $a_{i}$ for criterion $c_{j}$ given by expert $e_{\delta} . \bar{S}=\left\{s_{0}, s_{1}, \ldots, s_{t-1}\right\}$ is a predefined linguistic term set.

6.1. The Model Based on GRA Method to Obtain the Weight Vector of Criteria. The GRA method as an important approach to obtain the criteria weights has been researched by many scholars $[39,40]$. In what follows, the model based on GRA method shall be established to obtain the optimal weight vector of criteria.

Assume that $R=\left[r_{i j}\right]_{m \times n}$ is the collective Pythagorean fuzzy uncertain linguistic matrix, where $r_{i j}=\left\langle\left[s_{\theta_{i j}}, s_{\tau_{i j}}\right],\left(u_{i j}\right.\right.$, $\left.\left.v_{i j}\right)\right\rangle(i=1,2, \ldots, m ; j=1,2, \ldots, n)$. Let $\widetilde{R}^{+}=\left(\widetilde{r}_{1}^{+}, \widetilde{r}_{2}^{+}, \ldots\right.$, $\left.\widetilde{r}_{n}^{+}\right)$and $\widetilde{R}^{-}=\left(\widetilde{r}_{1}^{-}, \widetilde{r}_{2}^{-}, \ldots, \widetilde{r}_{n}^{-}\right)$denote the positive and negative ideal alternatives, respectively, where

$$
\begin{aligned}
& \tilde{r}_{j}^{+}=\left\langle\left[\max _{1 \leq i \leq m} s_{\theta_{i j}}, \max _{1 \leq i \leq m} s_{\tau_{i j}}\right],\left(\max _{1 \leq i \leq m} u_{i j}, \min _{1 \leq i \leq m} v_{i j}\right)\right\rangle ; \\
& \tilde{r}_{j}=\left\langle\left[\min _{1 \leq i \leq m} s_{\theta_{i j}}, \min _{1 \leq i \leq m} s_{\tau_{i j}}\right],\left(\min _{1 \leq i \leq m} u_{i j}, \max _{1 \leq i \leq m} v_{i j}\right)\right\rangle .
\end{aligned}
$$

The grey relational coefficient of each solution from positive ideal alternative and negative ideal alternative is obtained by utilizing the following equations, respectively:

$$
\begin{aligned}
& \xi_{i j}^{+} \\
& =\frac{\min _{1 \leq i \leq m} \min _{1 \leq j \leq n} d\left(r_{i j}, \tilde{r}_{j}^{+}\right)+\rho \max _{1 \leq i \leq m} \max _{1 \leq j \leq n} d\left(r_{i j}, \widetilde{r}_{j}^{+}\right)}{d\left(r_{i j}, \widetilde{r}_{j}^{+}\right)+\rho \max _{1 \leq i \leq m} \max _{1 \leq j \leq n} d\left(r_{i j}, \widetilde{r}_{j}^{+}\right)} ; \\
& \xi_{i j}^{-} \\
& =\frac{\min _{1 \leq i \leq m} \min _{1 \leq j \leq n} d\left(r_{i j}, \widetilde{r}_{j}^{-}\right)+\rho \max _{1 \leq i \leq m} \max _{1 \leq j \leq n} d\left(r_{i j}, \widetilde{r}_{j}^{-}\right)}{d\left(r_{i j}, \widetilde{r}_{j}^{-}\right)+\rho \max _{1 \leq i \leq m} \max _{1 \leq j \leq n} d\left(r_{i j}, \widetilde{r}_{j}^{-}\right)},
\end{aligned}
$$

for all $i=1,2, \ldots, m$ and $j=1,2, \ldots, n$, where the identification coefficient $\rho=0.5$ and the distance is normalized Hamming distance between PFULVs.

If the information about criteria weight is completely unknown, the single objective programming model is established to obtain the weight information.

$$
\begin{array}{ll}
\min & \sum_{i=1}^{m} \sum_{j=1}^{n}\left(\left(1-\xi_{i j}^{+}\right) \omega_{j}\right)^{2}+\left(\xi_{i j}^{-} \omega_{j}\right)^{2} \\
\text { s.t. } & \sum_{j=1}^{n} \omega_{j}=1 \\
& \omega_{j} \geq 0 .
\end{array}
$$

By solving the above model, we can obtain the optimal solution $\omega=\left(\omega_{1}, \omega_{2}, \ldots, \omega_{n}\right)^{T}$, which can be utilized as the weight vector of criteria.

6.2. The Decision Procedure. Based on established model and presented operators, we propose a procedure to solve the MCGDM problems in which the criteria values are in the form of PFULVs, and the information about the criteria weights is completely unknown. The detailed decisionmaking steps are listed as follows.

Step 1. Utilize formula (77) to calculate the values of $T_{i j}^{\delta}(\delta=$ $1,2, \ldots, q)$.

$$
\begin{aligned}
& T_{i j}^{1}=1, \\
& T_{i j}^{\delta}=\prod_{l=1}^{\delta-1} S\left(r_{i j}^{l}\right) \quad(\delta=2,3, \ldots, q) .
\end{aligned}
$$

Step 2. Utilize PFULPWAA operator in formula (78) or PFULPWGA operator in formula (79) to aggregate the evaluation information of individual expert to collective information.

$$
\begin{aligned}
& r_{i j}=\left\langle\left[s_{\theta_{i j}}, s_{\tau_{i j}}\right],\left(u_{i j}, v_{i j}\right)\right\rangle=\operatorname{PFULWAA}\left(r_{i j}^{1}, r_{i j}^{2}, \ldots,\right. \\
& \left.r_{i j}^{q}\right)=\left\langle\left[s_{\sum_{\delta=1}^{q}\left(T_{\delta} / \sum_{\delta=1}^{q} T_{\delta}\right) \theta_{i j}}, s_{\sum_{\delta=1}^{q}\left(T_{\delta} / \sum_{\delta=1}^{q} T_{\delta}\right) \tau_{i j}}\right],\right.
\end{aligned}
$$

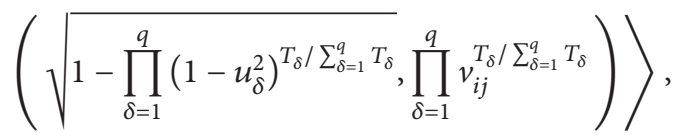

$$
\begin{aligned}
& i=1,2, \ldots, m ; j=1,2, \ldots, n ; \delta=1,2, \ldots, q,
\end{aligned}
$$




$$
\begin{gathered}
r_{i j}=\left\langle\left[s_{\theta_{i j}}, s_{\tau_{i j}}\right],\left(u_{i j}, v_{i j}\right)\right\rangle=\operatorname{PFULWGA}\left(r_{i j}^{1}, r_{i j}^{2}, \ldots,\right. \\
\left.r_{i j}^{q}\right)=\left\langle\left[ s_{\left.\prod_{\delta=1}^{q} \theta_{i j}^{T_{\delta} / \sum_{\delta=1}^{q} T_{\delta}}, s_{\prod_{\delta=1}^{q} \tau_{i j}^{T_{\delta}} / \sum_{\delta=1}^{q} T_{\delta}}\right],}\right.\right. \\
\left(\prod_{\delta=1}^{q} u_{i j}^{T_{\delta} / \sum_{\delta=1}^{q} T_{\delta}}, \sqrt{\left.\left.1-\prod_{\delta=1}^{q}\left(1-v_{\delta}^{2}\right)^{T_{\delta} / \sum_{\delta=1}^{q} T_{\delta}}\right)\right\rangle}\right. \\
i=1,2, \ldots, m ; j=1,2, \ldots, n ; \delta=1,2, \ldots, q .
\end{gathered}
$$

Step 3. Utilize formulas (80) to determine the positive and negative ideal alternatives, respectively.

$$
\begin{aligned}
& \widetilde{R}^{+}=\left(\widetilde{r}_{1}^{+}, \widetilde{r}_{2}^{+}, \ldots, \widetilde{r}_{n}^{+}\right), \\
& \widetilde{R}^{-}=\left(\widetilde{r}_{1}^{-}, \widetilde{r}_{2}^{-}, \ldots, \widetilde{r}_{n}^{-}\right),
\end{aligned}
$$

where

$$
\begin{array}{r}
\widetilde{r}_{j}^{+}=\left\langle\left[\max _{1 \leq i \leq m} s_{\theta_{i j}}, \max _{1 \leq i \leq m} s_{\tau_{i j}}\right],\left(\max _{1 \leq i \leq m} u_{i j}, \min _{1 \leq i \leq m} v_{i j}\right)\right\rangle, \\
j=1,2, \ldots, n, \\
\widetilde{r}_{j}^{-}=\left\langle\left[\min _{1 \leq i \leq m} s_{\theta_{i j}}, \min _{1 \leq i \leq m} s_{\tau_{i j}}\right],\left(\min _{1 \leq i \leq m} u_{i j}, \max _{1 \leq i \leq m} v_{i j}\right)\right\rangle, \\
j=1,2, \ldots, n .
\end{array}
$$

Step 4. Utilize formulas (82) to calculate the grey relational coefficient of each solution from positive ideal alternative and negative ideal alternative, respectively.

$$
\begin{aligned}
& \xi_{i j}^{+} \\
& =\frac{\min _{1 \leq i \leq m} \min _{1 \leq j \leq n} d\left(r_{i j}, \widetilde{r}_{j}^{+}\right)+\rho \max _{1 \leq i \leq m} \max _{1 \leq j \leq n} d\left(r_{i j}, \tilde{r}_{j}^{+}\right)}{d\left(r_{i j}, \widetilde{r}_{j}^{+}\right)+\rho \max _{1 \leq i \leq m} \max _{1 \leq j \leq n} d\left(r_{i j}, \widetilde{r}_{j}^{+}\right)}, \\
& i=1,2, \ldots, m ; j=1,2, \ldots, n \\
& \xi_{i j}^{-} \\
& \begin{array}{r}
=\frac{\min _{1 \leq i \leq m} \min _{1 \leq j \leq n} d\left(r_{i j}, \widetilde{r}_{j}^{-}\right)+\rho \max _{1 \leq i \leq m} \max _{1 \leq j \leq n} d\left(r_{i j}, \widetilde{r}_{j}^{-}\right)}{d\left(r_{i j}, \widetilde{r}_{j}^{-}\right)+\rho \max _{1 \leq i \leq m} \max _{1 \leq j \leq n} d\left(r_{i j}, \widetilde{r}_{j}^{-}\right)}, \\
i=1,2, \ldots, m ; j=1,2, \ldots, n,
\end{array}
\end{aligned}
$$

where the identification coefficient $\rho=0.5$ and the distance is normalized Hamming distance between PFULVs.

Step 5. Utilize model (83) to get the optimal weight vector of the criteria $\omega=\left(\omega_{1}, \omega_{2}, \ldots, \omega_{n}\right)^{T}$.

$$
\begin{aligned}
\min & \sum_{i=1}^{m} \sum_{j=1}^{n}\left(\left(1-\xi_{i j}^{+}\right) \omega_{j}\right)^{2}+\left(\xi_{i j}^{-} \omega_{j}\right)^{2} \\
\text { s.t. } & \sum_{j=1}^{n} \omega_{j}=1 \\
& \omega_{j} \geq 0 .
\end{aligned}
$$

Step 6. Utilize WPFULMSMA operator in formula (84) to aggregate the collective evaluation information of each criterion to the comprehensive evaluation information of each alternative.

$$
\begin{aligned}
& r_{i}=\left\langle\left[s_{\theta_{i}}, s_{\tau_{i}}\right],\left(u_{i}, v_{i}\right)\right\rangle=\operatorname{WPFULMSMA}^{(\kappa)}\left(r_{i 1}, r_{i 2}, \ldots, r_{i n}\right)=\left\langle s_{\left(\left(\sum_{1 \leq j_{1}<\cdots<j_{\kappa} \leq n} \prod_{l=1}^{\kappa} \omega_{j_{l}} \theta_{i j_{l}}\right) / C_{n}^{\kappa}\right)^{1 / \kappa}},\right. \\
& s_{\left.\left(\left(\sum_{1 \leq j_{1}<\cdots<j_{\kappa} \leq n} \prod_{l=1}^{\kappa} \omega_{j_{l}} \tau_{i_{l} l}\right) / C_{n}^{\kappa}\right)^{1 / \kappa}\right]},\left(\sqrt{\left(1-\left(\prod_{1 \leq j_{1}<\cdots<j_{\kappa} \leq n}\left(1-\prod_{l=1}^{\kappa}\left(1-\left(1-u_{i j_{l}}^{2}\right)^{\omega_{j_{l}}}\right)\right)\right)^{1 / C_{n}^{\kappa}}\right)^{1 / \kappa}},\right. \\
& \left.\left.\sqrt{1-\left(1-\left(\prod_{1 \leq j_{1}<\cdots<j_{\kappa} \leq n}\left(1-\prod_{l=1}^{\kappa}\left(1-v_{i j_{l}}^{2 \omega_{j_{l}}}\right)\right)\right)^{1 / C_{n}^{\kappa}}\right)^{1 / \kappa}}\right)\right\rangle, \quad i=1,2, \ldots, m ; \kappa=1,2, \ldots, n
\end{aligned}
$$

Step 7. Utilize formulas (85) to calculate the score values $S\left(r_{i}\right)$ and accuracy values $A\left(r_{i}\right)$ of each comprehensive evaluation value $r_{i}(i=1,2, \ldots, m)$, respectively.

$$
S\left(p_{i}\right)=\frac{\theta_{i}+\tau_{i}}{4(t-1)}\left(u_{i}^{2}+1-v_{i}^{2}\right), \quad i=1,2, \ldots, m,
$$

$$
A\left(r_{i}\right)=\frac{\theta_{i}+\tau_{i}}{2(t-1)}\left(u_{i}^{2}+v_{i}^{2}\right), \quad i=1,2, \ldots, m
$$

Step 8. Rank all the alternatives $\left\{a_{1}, a_{2}, \ldots, a_{n}\right\}$ according to Definition 8 , and select the most eligible alternative(s).

Step 9. End. 


\section{An Illustrative Example}

To demonstrate the application of the developed MCGDM method, we will cite an example from [24] which is about the investment selection. An investment company wanted to invest lots of money to develop a software project, and there are four possible projects $\left\{a_{1}, a_{2}, a_{3}, a_{4}\right\}$ as alternatives. In order to make a reasonable decision, three experts $\left\{e_{1}, e_{2}, e_{3}\right\}$ are designated to evaluate the alternatives with respect to three criteria: the technical feasibility $\left(c_{1}\right)$, the economic feasibility $\left(c_{2}\right)$, and the operational feasibility $\left(c_{3}\right)$. Suppose that there is a prioritization relationship for the experts, $e_{2}>e_{3}>e_{1}$, and the weight vector of three criteria is completely unknown. The experts gave the individual Pythagorean fuzzy uncertain linguistic decision matrices $R^{\delta}=\left[r_{i j}^{\delta}\right]_{4 \times 3}(\delta=1,2,3)$ by utilizing the linguistic term set $\bar{S}=\left\{s_{0}, s_{1}, s_{2}, s_{3}, s_{4}, s_{5}, s_{6}\right\}=$ (very poor, poor, slightly poor, fair, slightly good, good, very good) as listed in Tables 1-3.

7.1. The Evaluation Steps by Developed Method. To obtain the most eligible alternative(s), the detailed steps are shown as follows.

Step 1. Utilize formula (77) to calculate the values of $T_{i j}^{\delta}(\delta=$ $1,2,3)$.

$$
\begin{aligned}
T_{i j}^{1} & =\left(\begin{array}{lll}
1 & 1 & 1 \\
1 & 1 & 1 \\
1 & 1 & 1 \\
1 & 1 & 1
\end{array}\right), \\
T_{i j}^{2} & =\left(\begin{array}{lll}
0.3767 & 0.1883 & 0.3000 \\
0.1292 & 0.3500 & 0.4233 \\
0.2200 & 0.4000 & 0.3500 \\
0.3000 & 0.2317 & 0.6667
\end{array}\right), \\
T_{i j}^{3} & =\left(\begin{array}{lll}
0.0838 & 0.0399 & 0.1800 \\
0.0383 & 0.1356 & 0.0564 \\
0.0444 & 0.1480 & 0.1412 \\
0.1240 & 0.0436 & 0.2417
\end{array}\right) .
\end{aligned}
$$

Step 2. Utilize the PFULPWAA operator in formula (78) to aggregate all the individual decision matrices $R^{\delta}=$ $\left[r_{i j}^{\delta}\right]_{m \times n}(\delta=1,2,3)$ into the collective decision matrix $R=$ $\left[r_{i j}\right]_{m \times n}$, which is listed in Table 4.

Step 3. Utilize formulas (80) to determine the positive and negative ideal alternatives, respectively.

$$
\begin{gathered}
\widehat{R}^{+}=\left(\left\langle\left[s_{2.6273}, s_{4.6273}\right],(0.7779,0.2000)\right\rangle,\right. \\
\left\langle\left[s_{2.5504}, s_{4.5504}\right],(0.7822,0.3000)\right\rangle, \\
\left.\left\langle\left[s_{3.1747}, s_{5.1747}\right],(0.7906,0.2000)\right\rangle\right),
\end{gathered}
$$

TABle 1: Decision matrix $R^{1}$.

\begin{tabular}{cccc}
\hline & $c_{1}$ & $c_{2}$ & $c_{3}$ \\
\hline$a_{1}$ & $\left\langle\left[s_{1}, s_{3}\right],(0.8,0.6)\right\rangle$ & $\left\langle\left[s_{2}, s_{4}\right],(0.7,0.3)\right\rangle$ & $\left\langle\left[s_{3}, s_{5}\right],(0.6,0.2)\right\rangle$ \\
$a_{2}$ & $\left\langle\left[s_{2}, s_{4}\right],(0.7,0.6)\right\rangle$ & $\left\langle\left[s_{3}, s_{5}\right],(0.8,0.3)\right\rangle$ & $\left\langle\left[s_{1}, s_{3}\right],(0.4,0.2)\right\rangle$ \\
$a_{3}$ & $\left\langle\left[s_{3}, s_{5}\right],(0.6,0.2)\right\rangle$ & $\left\langle\left[s_{1}, s_{3}\right],(0.6,0.4)\right\rangle$ & $\left\langle\left[s_{2}, s_{4}\right],(0.8,0.2)\right\rangle$ \\
$a_{4}$ & $\left\langle\left[s_{1}, s_{3}\right],(0.7,0.4)\right\rangle$ & $\left\langle\left[s_{0}, s_{2}\right],(0.7,0.5)\right\rangle$ & $\left\langle\left[s_{3}, s_{5}\right],(0.9,0.2)\right\rangle$ \\
\hline
\end{tabular}

TABLE 2: Decision matrix $R^{2}$.

\begin{tabular}{cccc}
\hline & $c_{1}$ & $c_{2}$ & $c_{3}$ \\
\hline$a_{1}$ & $\left\langle\left[s_{3}, s_{5}\right],(0.7,0.6)\right\rangle$ & $\left\langle\left[s_{1}, s_{3}\right],(0.7,0.6)\right\rangle$ & $\left\langle\left[s_{2}, s_{4}\right],(0.6,0.4)\right\rangle$ \\
$a_{2}$ & $\left\langle\left[s_{0}, s_{2}\right],(0.8,0.3)\right\rangle$ & $\left\langle\left[s_{2}, s_{4}\right],(0.7,0.3)\right\rangle$ & $\left\langle\left[s_{3}, s_{5}\right],(0.6,0.3)\right\rangle$ \\
$a_{3}$ & $\left\langle\left[s_{1}, s_{3}\right],(0.6,0.2)\right\rangle$ & $\left\langle\left[s_{3}, s_{5}\right],(0.6,0.4)\right\rangle$ & $\left\langle\left[s_{2}, s_{4}\right],(0.7,0.3)\right\rangle$ \\
$a_{4}$ & $\left\langle\left[s_{2}, s_{4}\right],(0.6,0.4)\right\rangle$ & $\left\langle\left[s_{1}, s_{3}\right],(0.8,0.5)\right\rangle$ & $\left\langle\left[s_{4}, s_{6}\right],(0.8,0.2)\right\rangle$ \\
\hline
\end{tabular}

TABLE 3: Decision matrix $R^{3}$.

\begin{tabular}{cccc}
\hline & $c_{1}$ & $c_{2}$ & $c_{3}$ \\
\hline$a_{1}$ & $\left\langle\left[s_{2}, s_{4}\right],(0.5,0.6)\right\rangle$ & $\left\langle\left[s_{1}, s_{3}\right],(0.6,0.3)\right\rangle$ & $\left\langle\left[s_{6}, s_{6}\right],(0.6,0.4)\right\rangle$ \\
$a_{2}$ & $\left\langle\left[s_{3}, s_{5}\right],(0.5,0.6)\right\rangle$ & $\left\langle\left[s_{2}, s_{4}\right],(0.8,0.3)\right\rangle$ & $\left\langle\left[s_{0}, s_{2}\right],(0.8,0.2)\right\rangle$ \\
$a_{3}$ & $\left\langle\left[s_{1}, s_{3}\right],(0.5,0.2)\right\rangle$ & $\left\langle\left[s_{2}, s_{4}\right],(0.8,0.4)\right\rangle$ & $\left\langle\left[s_{3}, s_{5}\right],(0.5,0.2)\right\rangle$ \\
$a_{4}$ & $\left\langle\left[s_{3}, s_{5}\right],(0.7,0.5)\right\rangle$ & $\left\langle\left[s_{1}, s_{3}\right],(0.7,0.6)\right\rangle$ & $\left\langle\left[s_{2}, s_{4}\right],(0.7,0.2)\right\rangle$ \\
\hline
\end{tabular}

$$
\begin{gathered}
\widehat{R}^{-}=\left(\left\langle\left[s_{0.3976}, s_{2.3976}\right],(0.5849,0.6000)\right\rangle,\right. \\
\left\langle\left[s_{0.9768}, s_{2.9658}\right],(0.6696,0.5275)\right\rangle \\
\left.\left\langle\left[s_{2.0655}, s_{4.0655}\right],(0.6000,0.3677)\right\rangle\right) .
\end{gathered}
$$

Step 4. Utilize formulas (82) to calculate the grey relational coefficient of each solution from positive ideal alternative and negative ideal alternative, respectively (suppose $\rho=0.5$ )

$$
\begin{aligned}
& {\left[\xi_{i j}^{+}\right]_{4 \times 3}=\left(\begin{array}{lll}
0.3333 & 0.3661 & 0.3573 \\
0.3346 & 0.6594 & 0.4507 \\
0.3905 & 0.5000 & 0.4690 \\
0.4514 & 0.3677 & 1.0000
\end{array}\right),} \\
& {\left[\xi_{i j}^{-}\right]_{4 \times 3}=\left(\begin{array}{lll}
0.3709 & 1.0000 & 0.6974 \\
0.6707 & 0.4466 & 0.7404 \\
0.4721 & 0.4436 & 0.7198 \\
0.4164 & 0.7059 & 0.4084
\end{array}\right)}
\end{aligned}
$$

Step 5. Utilize model (83) to obtain the optimal weight vector of the criteria.

$$
\begin{array}{ll}
\min & 2.5434 \omega_{1}^{2}+3.0622 \omega_{2}^{2}+2.7163 \omega_{3}^{2} \\
\text { s.t. } & \sum_{j=1}^{3} \omega_{j}=1, \\
& \omega_{j} \geq 0 \\
& (j=1,2,3) .
\end{array}
$$


TABLE 4: Collective decision matrix $R$.

\begin{tabular}{llll}
\hline & $c_{1}$ & $c_{2}$ & $c_{3}$ \\
\hline$a_{1}$ & $\left\langle\left[s_{2.6237}, s_{4.6273}\right],(0.6692,0.6000)\right\rangle$ & $\left\langle\left[s_{1.0325}, s_{3.0325}\right],(0.6870,0.5275)\right\rangle$ & $\left\langle\left[s_{2.9324}, s_{4.5270}\right],(0.6000,0.3677)\right\rangle$ \\
$a_{2}$ & $\left\langle\left[s_{0.3976}, s_{2.3976}\right],(0.7779,0.3314)\right\rangle$ & $\left\langle\left[s_{2.0913}, s_{4.0913}\right],(0.7382,0.3000)\right\rangle$ & $\left\langle\left[s_{2.0655}, s_{4.0655}\right],(0.6719,0.2630)\right\rangle$ \\
$a_{3}$ & $\left\langle\left[s_{1.0702}, s_{3.0702}\right],(0.5849,0.2000)\right\rangle$ & $\left\langle\left[s_{2.5504}, s_{4.5504}\right],(0.6696,0.4000)\right\rangle$ & $\left\langle\left[s_{2.2347}, s_{4.2347}\right],(0.6781,0.2625)\right\rangle$ \\
$a_{4}$ & $\left\langle\left[s_{2.1236}, s_{4.1236}\right],(0.6339,0.4193)\right\rangle$ & $\left\langle\left[s_{0.9658}, s_{2.9658}\right],(0.7822,0.5168)\right\rangle$ & $\left\langle\left[s_{3.1747}, s_{5.1747}\right],(0.7906,0.2000)\right\rangle$ \\
\hline
\end{tabular}

Solving this model by Lingo, we obtain

$$
\begin{aligned}
& \omega_{1}=0.3614, \\
& \omega_{2}=0.3002, \\
& \omega_{3}=0.3384 .
\end{aligned}
$$

Step 6. Utilize the WPFULMSMA operator in formula (84) to aggregate the collective evaluation values $r_{i j}(i=$ $1,2,3,4 ; j=1,2,3)$ in $i$ th of $R$ to obtain the comprehensive evaluation values $r_{i}(i=1,2,3,4)($ suppose $\kappa=2)$

$$
\begin{aligned}
& r_{1}=\left\langle\left[s_{0.7174}, s_{1.3514}\right],(0.4106,0.7944)\right\rangle, \\
& r_{2}=\left\langle\left[s_{0.4580}, s_{1.1469}\right],(0.4739,0.6687)\right\rangle, \\
& r_{3}=\left\langle\left[s_{0.6239}, s_{1.2991}\right],(0.4043,0.6584)\right\rangle, \\
& r_{4}=\left\langle\left[s_{0.6729}, s_{1.3535}\right],(0.4805,0.7220)\right\rangle .
\end{aligned}
$$

Step 7. Utilize formulas (85) to calculate the score values $S\left(r_{i}\right)$ and accuracy values $A\left(r_{i}\right)$ of each comprehensive evaluation value $r_{i}(i=1,2, \ldots, m)$, respectively.

$$
\begin{aligned}
& S\left(r_{1}\right)=0.0463 \\
& S\left(r_{2}\right)=0.0520 \\
& S\left(r_{3}\right)=0.0585 \\
& S\left(r_{4}\right)=0.0599 \\
& A\left(r_{1}\right)=0.1379 \\
& A\left(r_{2}\right)=0.0898 \\
& A\left(r_{3}\right)=0.0957 \\
& A\left(r_{4}\right)=0.1270
\end{aligned}
$$

Step 8. Rank all the alternatives $\left\{a_{1}, a_{2}, \ldots, a_{n}\right\}$ according to Definition 8, and select the most eligible alternative(s).

$$
a_{4}>a_{3}>a_{2}>a_{1} .
$$

Thus, the best alternative is $a_{4}$.

7.2. The Effect of the Parameter $\kappa$ on the Ranking Result of This Example. To demonstrate the effect of the parameter $\kappa$ on the ranking result of this example, we can utilize the different values of $\kappa$ in Step 6 to rank the alternatives. The ranking results are listed in Table 5.
TABLE 5: Ordering of the alternatives by utilizing different $\kappa$.

\begin{tabular}{lcc}
\hline$\kappa$ & Score value $S\left(r_{i}\right)(i=1,2,3,4)$ & Ranking \\
\hline \multirow{4}{*}{$S\left(r_{1}\right)=0.0485}$, & \\
& $S\left(r_{2}\right)=0.0537$, & $a_{4}>a_{3}>a_{2}>a_{1}$ \\
& $S\left(r_{3}\right)=0.0603$, & \\
& $S\left(r_{4}\right)=0.0644$. & \\
& $S\left(r_{1}\right)=0.0463$, & \\
& $S\left(r_{2}\right)=0.0520$, & $a_{4}>a_{3}>a_{2}>a_{1}$ \\
& $S\left(r_{3}\right)=0.0585$, & \\
& $S\left(r_{4}\right)=0.0599$. & \\
& $S\left(r_{1}\right)=0.0441$, & \\
& $S\left(r_{2}\right)=0.0495$, & \\
& $S\left(r_{3}\right)=0.0571$, & \\
& $S\left(r_{4}\right)=0.0562$. & \\
\hline
\end{tabular}

Clearly, the ordering may be slightly different for the different aggregation parameter $\kappa$. However, the best alternative is $a_{4}$ or $a_{3}$. In addition, by further analysis, we can see that the score values got by the WPFULMSMA operator become smaller as the parameter $\kappa$ increases for the same integrated elements. The value of the parameter $\kappa$ can be utilized to reflect the risk preferences of expert; the larger the parameter $\kappa$ is, the more risk preferences the expert has. On the contrary, the smaller the parameter $\kappa$ is, the more risk averse the expert is. Generally speaking, if the expert does not have special preferences, he/she can consider the ranking result of the parameter $\kappa$ getting [ $n / 2$ ], where symbol [] denotes round function and $n$ is the number of criteria, which is not only simple and intuitive, but in this situation, the risk preferences of expert are neutral and the interrelationship among the input arguments can be globally considered.

7.3. Comparison with the Existing Method. This example is obtained from [24], so we can directly compare with it. From the ranking results of the alternatives, there are two ranking in [24], which are $a_{4}>a_{3}>a_{2}>a_{1}$ and $a_{4}>a_{1}>a_{3}>a_{2}$, respectively. When $\kappa=1$ or $\kappa=2$, we can also obtain the same ranking result $a_{4}>a_{3}>a_{2}>a_{1}$. This will show the effectiveness of the proposed method. The advantages of the developed method in the paper are shown as follows:

(1) PFULV has virtue of being more precise and practical than PFLV. The PFULV can better describe uncertainty and retain the original data integrity or the experts' inherent thoughts, which is the precondition of ensuring precision of final results.

(2) The weights of experts, which are determined by the PFULPWAA operator based on the prioritization 
among the experts, are more objective and reasonable than the known weight vector of experts.

(3) The Pythagorean fuzzy linguistic weighted averaging (PFLWA) operator and extension of TOPSIS in [24] are based on the assumption that the input arguments are independent. However, the PFULPWAA and PFULPWGA operators can consider prioritization among the experts or criteria, and the WPFULMSMA operator can consider the interrelationship among the multi-input arguments, which could ensure the reasonableness and effectiveness of the decision-making results.

(4) The WPFILMSMA operator provides more selecting choice for experts by altering the values of the parameter decided by the preferences of the expert, which is more flexible for coping with MCGDM problems with PFULVS.

\section{Conclusion}

To solve more complex MCGDM problems, we define a new class of fuzzy set called PFULS as an extension of PFLS, which could more precisely express ULV and retain the original data integrity. The operational laws, score function, accuracy function, and normalized Hamming distance of PFULVs are presented. Then, based on the PA operator, we propose the PFULPWAA and PFULPWGA operators, which can capture the prioritization among the experts or criteria. In addition, we combine MSM with PFULVs and propose the PFULMSMA and WPFULMSMA operators, which can consider the interrelationship among the multiinput arguments. In the meantime, some special cases and properties are also investigated, such as the idempotency, commutativity, monotonicity, and boundedness. Furthermore, a Pythagorean fuzzy uncertain linguistic MCGDM method based on GRA model and proposed operators with interactive condition and completely unknown weight information is developed. The prominent characteristics of developed method are that they are able to effectively aggregate PFULVS, and they can capture the prioritization among the experts and interrelationship among the multiinput arguments, which can avoid losing and distorting the given preference information so as to make the final results more feasible and practical. Finally, an example is provided to illustrate the feasibility and practicality of the developed method. In the succeeding work, the proposed operators shall be applied to the practical problems.

\section{Disclosure}

This article does not contain any studies with human participants or animals performed by any of the authors.

\section{Competing Interests}

The authors declare that they do not have any commercial or associative interests that represent a conflict of interests in connection with this manuscript. There are no professional or other personal interests that can inappropriately influence their submitted work.

\section{Acknowledgments}

This paper is supported by the National Natural Science Foundation of China (nos. 61273230, 61603011, 61603010, and 71471172), New Century Excellent Talents of 2012 (no. NCET-12-1027), Optimization of the Financial Industry and Regional Development Management Collaborative Innovation Center Project in Shandong Province (no. 14AWTJ014), Capital Social Construction and Social Management Collaborative Innovation Center Project, Postdoctoral Science Foundation of China (no. 2015M580940), and Postdoctoral Science Foundation of Beijing (no. 2016ZZ-10). This paper is also supported by the Special Funds of Taishan Scholars Project of Shandong Province.

\section{References}

[1] K. T. Atanassov, "Intuitionistic fuzzy sets," Fuzzy Sets and Systems, vol. 20, no. 1, pp. 87-96, 1986.

[2] K. T. Atanassov, G. Pasi, and R. R. Yager, "Intuitionistic fuzzy interpretations of multi-criteria multi-person and multimeasurement tool decision making," International Journal of Systems Science, vol. 36, no. 14, pp. 859-868, 2005.

[3] J. Qin and X. Liu, "An approach to intuitionistic fuzzy multiple attribute decision making based on Maclaurin symmetric mean operators," Journal of Intelligent \& Fuzzy Systems, vol. 27, no. 5, pp. 2177-2190, 2014.

[4] S.-M. Chen, S.-H. Cheng, and T.-C. Lan, "A novel similarity measure between intuitionistic fuzzy sets based on the centroid points of transformed fuzzy numbers with applications to pattern recognition," Information Sciences, vol. 343-344, pp. 1540, 2016.

[5] B.-B. Qiu, J. N. K. Liu, and W.-M. Ma, "Novel methods for intuitionistic fuzzy multiple attribute decision making," Journal of Software, vol. 7, no. 11, pp. 2553-2559, 2012.

[6] M. Montajabiha, "An extended PROMETHE II multi-criteria group decision making technique based on intuitionistic fuzzy logic for sustainable energy planning," Group Decision \& Negotiation, vol. 25, no. 2, pp. 221-244, 2016.

[7] Y. D. He, Z. He, and H. Huang, "Decision making with the generalized intuitionistic fuzzy power interaction averaging operators," Soft Computing, 2015.

[8] Y. He, H. Chen, Z. He, and L. Zhou, "Multi-attribute decision making based on neutral averaging operators for intuitionistic fuzzy information," Applied Soft Computing Journal, vol. 27, pp. 64-76, 2015.

[9] K. T. Atanassov, Intuitionistic Fuzzy Sets, vol. 35 of Studies in Fuzziness and Soft Computing, Springer, Heidelberg, Germany, 1999.

[10] K. T. Atanassov, "Geometrical interpretation of the elements of the intuitionistic fuzzy objects," International Journal Bioautomation, vol. 20, no. 1, pp. S27-S42, 2016.

[11] R. R. Yager, "Pythagorean fuzzy subsets," in Proceedings of the Joint IFSA World Congress and NAFIPS Annual Meeting, pp. 5761, Edmonton, Canada, 2013.

[12] R. R. Yager, "Pythagorean membership grades in multicriteria decision making," IEEE Transactions on Fuzzy Systems, vol. 22, no. 4, pp. 958-965, 2014. 
[13] X. Zhang, "A novel approach based on similarity measure for pythagorean fuzzy multiple criteria group decision making," International Journal of Intelligent Systems, vol. 31, no. 6, pp. 593611, 2016.

[14] X. L. Zhang and Z. S. Xu, "Extension of TOPSIS to multiple criteria decision making with pythagorean fuzzy sets," International Journal of Intelligent Systems, vol. 29, no. 12, pp. 1061-1078, 2014.

[15] P. Ren, Z. Xu, and X. Gou, "Pythagorean fuzzy TODIM approach to multi-criteria decision making," Applied Soft Computing Journal, vol. 42, pp. 246-259, 2016.

[16] X. D. Peng and Y. Yang, "Pythagorean fuzzy choquet integral based MABAC method for multiple attribute group decision making," International Journal of Intelligent Systems, vol. 31, no. 10, pp. 989-1020, 2016.

[17] Z. Tao, X. Liu, H. Chen, and J. Liu, "Entropy measures for linguistic information and its application to decision making," Journal of Intelligent \& Fuzzy Systems, vol. 29, no. 2, pp. 747-759, 2015.

[18] X. Liu, J. Zhu, G. Liu, and J. Hao, "A multiple attribute decision making method based on uncertain linguistic Heronian mean," Mathematical Problems in Engineering, vol. 2013, Article ID 597671, 11 pages, 2013.

[19] G. W. Wei, X. F. Zhao, R. Lin, and H. J. Wang, "Uncertain linguistic Bonferroni mean operators and their application to multiple attribute decision making," Applied Mathematical Modelling, vol. 37, no. 7, pp. 5277-5285, 2013.

[20] P. D. Liu and F. Jin, "Methods for aggregating intuitionistic uncertain linguistic variables and their application to group decision making," Information Sciences, vol. 205, pp. 58-71, 2012.

[21] Y. Ju, X. Liu, and D. Ju, "Some new intuitionistic linguistic aggregation operators based on Maclaurin symmetric mean and their applications to multiple attribute group decision making," Soft Computing, vol. 20, no. 11, pp. 4521-4548, 2016.

[22] P. Liu and L. Shi, "Intuitionistic uncertain linguistic powered Einstein aggregation operators and their application to multiattribute group decision making," The Journal of Applied Anal$y$ sis and Computation, vol. 5, no. 4, pp. 534-561, 2015.

[23] X. F. Wang and J. Q. Wang, "Approach to group decision making based on intuitionistic uncertain linguistic aggregation operators," Fuzzy Systems \& Operations Research and Management, vol. 367, pp. 223-232, 2015.

[24] X. D. Peng and Y. Yang, "Multiple attribute group decision making methods based on Pythagorean fuzzy linguistic set," Computer Engineering and Applications, vol. 52, no. 23, pp. 5054, 2016.

[25] R. R. Yager, “Prioritized aggregation operators," International Journal of Approximate Reasoning, vol. 48, no. 1, pp. 263-274, 2008.

[26] G. Wei, "Hesitant fuzzy prioritized operators and their application to multiple attribute decision making," Knowledge-Based Systems, vol. 31, pp. 176-182, 2012.

[27] X. F. Zhao, R. Lin, and G. W. Wei, "Fuzzy prioritized operators and their application to multiple attribute group decision making," Applied Mathematical Modelling, vol. 37, no. 7, pp. 4759-4770, 2013.

[28] X. H. Yu and Z. S. Xu, "Prioritized intuitionistic fuzzy aggregation operators," Information Fusion, vol. 14, no. 1, pp. 108-116, 2013.
[29] J. Ye, "Prioritized aggregation operators of trapezoidal intuitionistic fuzzy sets and their application to multicriteria decisionmaking," Neural Computing \& Applications, vol. 25, no. 6, pp. 1447-1454, 2014.

[30] X. Zhao, Q. Li, and G. Wei, "Some prioritized aggregating operators with linguistic information and their application to multiple attribute group decision making," Journal of Intelligent \& Fuzzy Systems, vol. 26, no. 4, pp. 1619-1630, 2014.

[31] Z. M. Zhang, "2-Tuple prioritized aggregation operators and their application to multiple attribute group decision making," British Journal of Mathematics \& Computer Science, vol. 26, no. 5, pp. 2185-2202, 2014.

[32] D.-H. Peng, T.-D. Wang, C.-Y. Gao, and H. Wang, "Multigranular uncertain linguistic prioritized aggregation operators and their application to multiple criteria group decision making," Journal of Applied Mathematics, vol. 2013, Article ID 857916, 13 pages, 2013.

[33] C. Maclaurin, "A second letter to Martin Folkes, Esq concerning the roots of equations, with demonstration of other rules of algebra," Philosophical Transactions of the Royal Society of London A, vol. 36, no. 407-416, pp. 59-96, 1729.

[34] J. Qin, X. Liu, and W. Pedrycz, "Hesitant fuzzy Maclaurin symmetric mean operators and its application to multiple-attribute decision making," International Journal of Fuzzy Systems, vol. 17, no. 4, pp. 509-520, 2015.

[35] Z. Zhang, F. Wei, and S. Zhou, "Approaches to comprehensive evaluation with 2-tuple linguistic information," International Journal of Fuzzy Systems, vol. 28, no. 1, pp. 469-475, 2014.

[36] J. Qin and X. Liu, "Approaches to uncertain linguistic multiple attribute decision making based on dual Maclaurin symmetric mean," Journal of Intelligent \& Fuzzy Systems, vol. 29, no. 1, pp. 171-186, 2015.

[37] Z. Xu, "Induced uncertain linguistic OWA operators applied to group decision making," Information Fusion, vol. 7, no. 2, pp. 231-238, 2006.

[38] J. Pečarić, J. Wen, W.-L. Wang, and T. Lu, "A generalization of Maclaurin's inequalities and its applications," Mathematical Inequalities \& Applications, vol. 8, no. 4, pp. 583-598, 2005.

[39] S. Ramesh, R. Viswanathan, and S. Ambika, "Measurement and optimization of surface roughness and tool wear via grey relational analysis, TOPSIS and RSA techniques," Measurement, vol. 78, pp. 63-72, 2016.

[40] H. X. Tang, "A novel fuzzy soft set approach in decision making based on grey relational analysis and Dempster-Shafer theory of evidence," Applied Soft Computing Journal, vol. 31, pp. 317-325, 2015. 


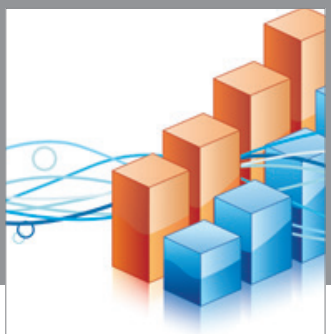

Advances in

Operations Research

vatem alat4

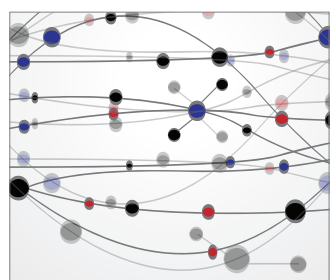

\section{The Scientific} World Journal
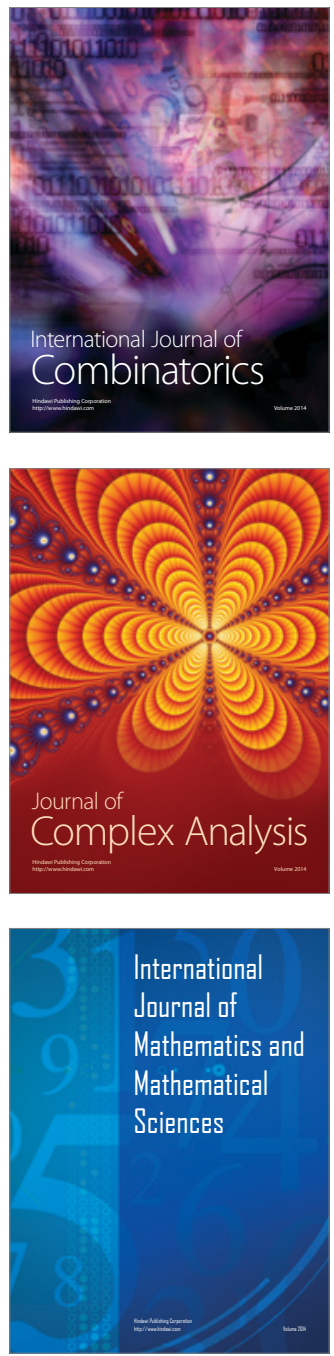
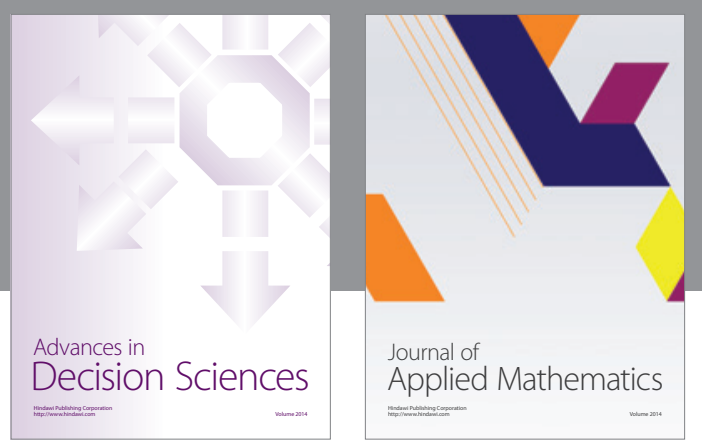

Algebra

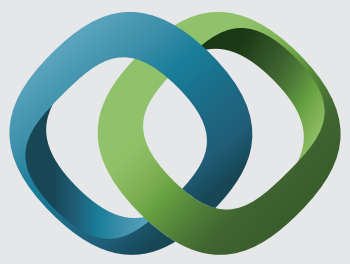

\section{Hindawi}

Submit your manuscripts at

https://www.hindawi.com
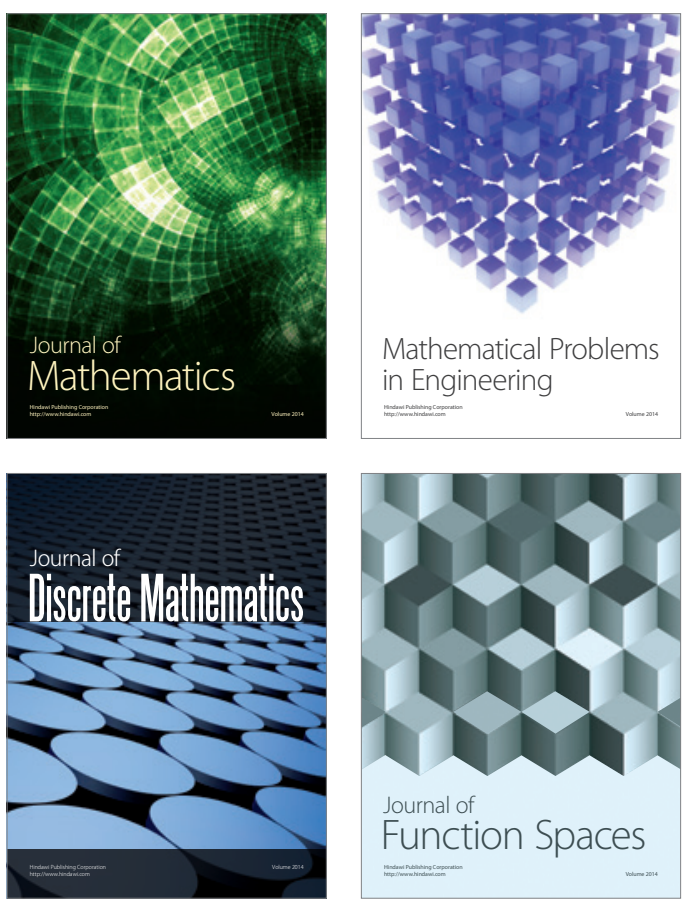

Mathematical Problems in Engineering
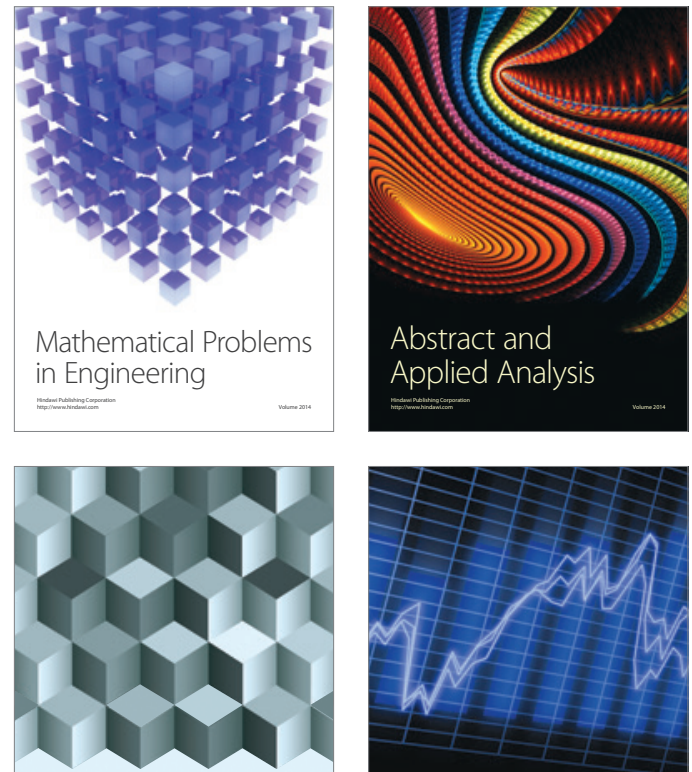

Journal of

Function Spaces

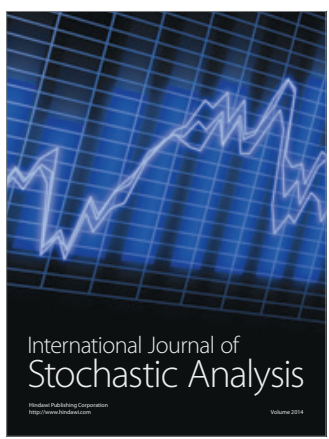

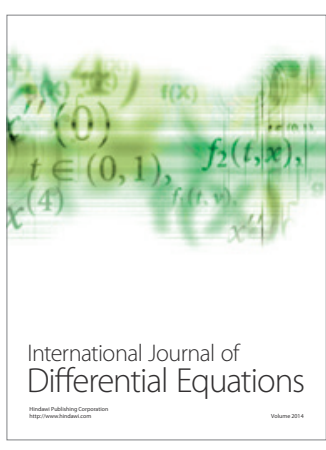
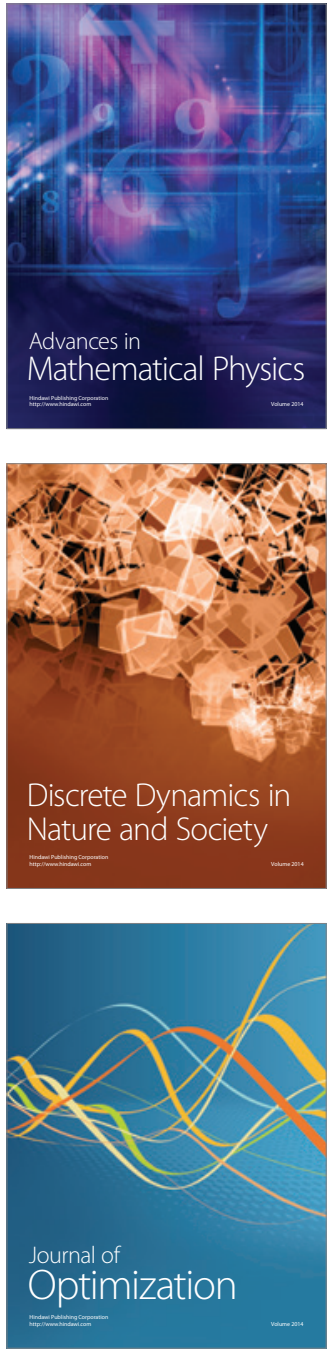